Mon. Not. R. Astron. Soc. 000, 000-000 (0000) Printed 16 October $2019 \quad$ (MN LATEX style file v2.2)

\title{
Detection of the nearest Jupiter analog in radial velocity and astrometry data
}

\author{
Fabo Feng ${ }^{1 \star}$, Guillem Anglada-Escudé2 ${ }^{2}$ Mikko Tuomi $^{3}$, Hugh R. A. Jones ${ }^{3}$, \\ Julio Chanamét,5, Paul R. Butler ${ }^{1}$, Markus Janson ${ }^{6}$ \\ ${ }^{1}$ Department of Terrestrial Magnetism, Carnegie Institution of Washington, 5241 Broad Branch Road, NW, Washington, DC 20015, USA \\ ${ }^{2}$ School of Physics and Astronomy, Queen Mary University of London, 327 Mile End Road, London, United Kingdom \\ ${ }^{3}$ Centre for Astrophysics Research, University of Hertfordshire, College Lane, AL10 9AB, Hatfield, UK \\ ${ }^{4}$ Instituto de Astrofísica, Pontificia Universidad Católica de Chile, Av. Vicuña Mackenna 4860, 782-0436 Macul, Santiago, Chile \\ ${ }^{5}$ Millennium Institute of Astrophysics, Santiago, Chile \\ ${ }^{6}$ Department of Astronomy,Stockholm University, AlbaNova University Center, 10691 Stockholm, Sweden
}

16 October 2019

\begin{abstract}
The presence of Jupiter is crucial to the architecture of the Solar System and models underline this to be a generic feature of planetary systems. We find the detection of the difference between the position and motion recorded by the contemporary astrometric satellite Gaia and its precursor Hipparcos can be used to discover Jupiter-like planets. We illustrate how observations of the nearby star $\epsilon$ Indi A giving astrometric and radial velocity data can be used to independently find the orbit of its suspected companion. The radial velocity and astrometric data provide complementary detections which allow for a much stronger solution than either technique would provide individually. We quantify $\epsilon$ Indi A b as the closest Jupiter-like exoplanet with a mass of $3 M_{\text {Jup }}$ on a slightly eccentric orbit with an orbital period of $45 \mathrm{yr}$. While other long-period exoplanets have been discovered, $\epsilon$ Indi A b provides a well constrained mass and along with the well-studied brown dwarf binary in orbit around $\epsilon$ Indi A means that the system provides a benchmark case for our understanding of the formation of gas giant planets and brown dwarfs.
\end{abstract}

Key words: methods: statistical - methods: data analysis - techniques: radial velocities - astrometry - stars: individual: $\epsilon$ Indi A

\section{INTRODUCTION}

One of the main goals of exoplanet searches is to understand our Solar System, with inner and outer planets including the benchmark planets: Earth and Jupiter. Although hot Jupiters around Sun-like stars and Earth-mass planets around M-dwarfs have been detected during the last decades (Gillon et al. 2016, Anglada-Escudé et al. 2016) few Jupiter-like planets have been detected around Sun-like stars and such detections when they are made have only been possible with a single technique (e.g., Kuzuhara et al. 2013, Wittenmyer et al. 2016).

$\epsilon$ Indi A is a good candidate to search for Solar System analogs. $\epsilon$ Indi A (HIP 108870, HR 8387, HD 209100, GJ 845) is a nearby $\mathrm{K} 2 \mathrm{~V}$ star $\left(3.62 \mathrm{pc}\right.$ according to van Leeuwen 2007) with a mass of $0.762 \pm 0.038 M_{\odot}$ (Demory et al. 2009), and a luminosity of $0.22 L_{\odot}$. This star is also accompanied by a binary brown dwarf with a separation of about 1459 au (Scholz et al. 2003). A clear long-term signal has been established in the radial velocity data by Endl et al. (2002) and Zechmeister et al. (2013). This signal is much larger than from the relatively distant brown dwarfs and supports the existence of another companion with a period longer than 30 years. The non-detection of this signal in direct imaging by Janson et al. (2009) suggests that the companion inducing the radial velocity signal has a relatively low temperature and a particularly interesting object for follow-up by multiple techniques.

^ E-mail: fengfabo@gmail.com 
To find small Keplerian signals and to investigate the previously proposed long period companion around $\epsilon$ Indi A, we analyze the data from Zechmeister et al. (2013) and the recent HARPS data in the ESO archive in the Bayesian framework. With new data and noise modelling techniques there is the potential for detection of weak signals (e.g. Feng et al. 2016, Feng, Tuomi \& Jones 2017b). Using the Agatha software (Feng, Tuomi \& Jones 2017a), we compare noise models to find the so-called Goldilocks model (Feng et al. 2016). We also calculate the Bayes factor periodograms (BFPs) to test the sensitivity of signals to the choice of noise models. These signals are further diagnosed by calculating the BFPs for various noise proxies and their residuals.

The combination of RV and astrometry has been used to detect and characterize a few short period planets, though no cold Jupiters have been detected in a similar way. Thanks to the two decades of baseline provided by the Hipparcos and Gaia survey (van Leeuwen 2007, Gaia Collaboration et al. 2018, Lindegren et al. 2018), we now have at least two epochs with well determined positions and proper motions, which are quite sensitive to the stellar reflex motion caused by Jupiter-like planets. Since the barycentric motion of the satellite is modeled in combination with proper motions in the analyses of the raw data (Lindegren et al. 2018), the astrometric survey data provides the position and velocity of a star at a given epoch viewed from the barycenter of the Solar System. $\epsilon$ Indi A provides a test case for a nearby object with a significant change in RV and change in position and proper motion between Hipparcos and Gaia over a long time baseline of observations.

This paper is structured as follows. First, we introduce the data in section 2 . Then we describe the statistical and numerical methods for the analysis of RV data and constrain the long period signal present in the data. In section 4 , we concentrate on the HARPS data and constrain the activity signals using posterior samplings. In section 5 , we analyze the RV and astrometry data to constrain the mass and orbital parameters of $\epsilon$ Indi A b. Finally, we discuss and conclude in section 6

\section{RV DATA AND MODEL}

We obtain the HARPS data by processing the spectra in the ESO archive (Programmes 60.A-9036, 072.C-0488, 072.C-0513, 073.C-0784, 074.C-0012, 077.C-0530, 078.C-0833, 079.C-0681, 081.D-0870, 183.C-0972, 087.D-0511, 091.C-0853, 091.C-0844, 094.C-0894, 192.C-0852, 196.C-1006, 098.C-0446) using the TERRA algorithm (Anglada-Escudé \& Butler 2012). The HARPS data also include various noise proxies courtesy of the HARPS pipeline, including Bisector span (BIS) and full width half maximum of spectra lines (FWHM). These are supplemented by TERRA-generated indices including $\mathrm{R}_{\mathrm{HK}}^{\prime}$ (or CaHK index), intensity of the H-alpha line (H-alpha), indices from sodium lines (NaD1 and NaD2), and the differential RV sets which record wavelength dependent noise (Feng, Tuomi \& Jones 2017b). We only use the 3AP3-2 differential set which is found to be correlated with RVs.

In the left-hand panel of Fig. 1] we show all the HARPS RVs including high cadence epochs (called "HARPS"; 4198 points). We note that the RVs from JD2455790 to JD2455805 are measured with high cadence, leading to 3636 RVs spread over two weeks. Such high-cadence data were obtained to study high frequency stellar oscillations and most of them are measured with a considerably lower signal to noise ratio than the rest of the data. To remove the stellar and instrumental systematics, we define the HARPSlow data set by excluding high cadence RVs with signal to noise ratio less than 110 (HARPShigh; shown by the grey points in Fig. 1). This dataset consists of 518 points. We are aware of the ill-determined offset for post-2015 data (Lo Curto et al. 2015) due to fibre change, which also give relatively high BIS (see the right panel of Fig. 1). We name the pre-2015 data set as "HARPSpre", and the post-2015 data as "HARPSpost". Thus the HARPS set is a combination of HARPSlow and HARPShigh while the HARPSlow is a combination of HARPSpre and HARPSpost. We will use these sub-sets to test the sensitivity of signals to the choice of dataset 1 in section 4.

An offset parameter is needed to combine HARPSpre and HARPSpost. To avoid potential degeneracy between this offset and Keplerian signals especially with period comparable with the HARPSpost time span, we use HARPSpre to investigate short-period signals. To constrain the long period signal, we use HARPSpre, HARPSpost as well as the CES long camera (LC) and very long camera (VLC) data sets from Zechmeister et al. (2013). We also use the UVES/VLT RVs which are measured at epochs of 2453272, 2457905, and 2457993 to constrain the trend. The UVES data is shown in Tables C1, C2, and C3. The other data sets are available at http://star.herts.ac.uk/ $\mathrm{hraj} / \mathrm{HD} 209100 /$.

To model the RV variation caused by stellar activity and planetary perturbations, we use a combined moving average (MA) and Keplerian model:

$$
\hat{v}_{r}\left(t_{i}\right)=v_{r}^{p}\left(t_{i}\right)+\sum_{l=1}^{q} w_{l} \exp \left(-\frac{\left|t_{i}-t_{i-k}\right|}{\tau}\right),
$$

where $\left\{w_{l}\right\}$ and $\tau$ are respectively the amplitude and timescale of the $q^{\text {th }}$ order MA model (or MA(q)). The planet-related RV

\footnotetext{
1 All of these data sets are available at http://star-www.herts.ac.uk/ ffeng/research_note/eps_indi/
} 

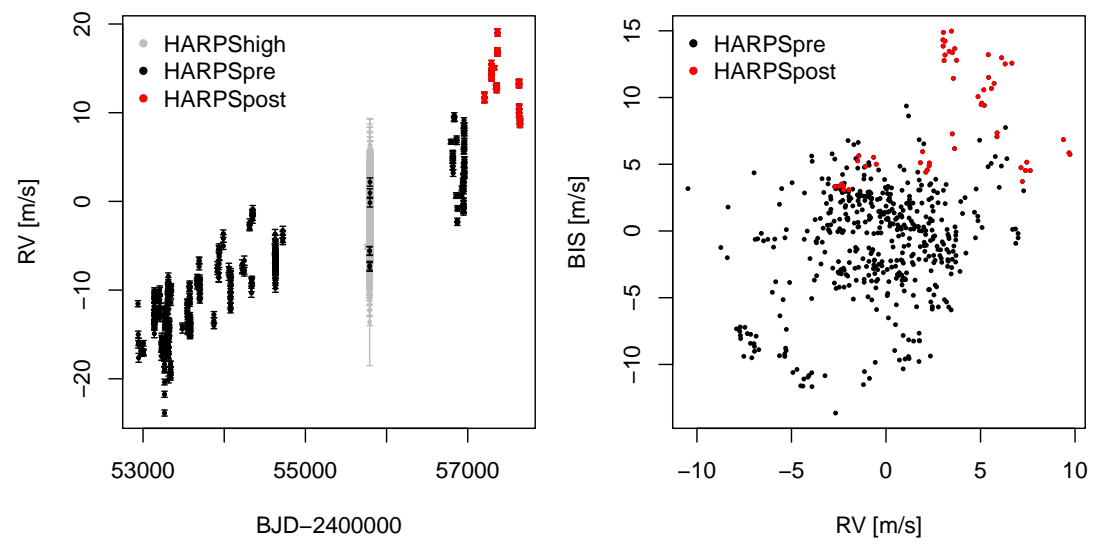

Figure 1. HARPS data of $\epsilon$ Indi A. Left panel: the HARPS data set consists of the high cadence RVs (HARPShigh) with signal to noise ratio less than 110 (grey) and the low cadence data (HARPSlow). The HARPSlow set includes the pre-2015 (HARPSpre; black) and post2015 (HARPSpost; red) RVs. Right panel: correlation between BISs and RVs of the HARPSlow set after subtraction by corresponding best-fitting parabola.

variation is

$$
v_{r}^{p}\left(t_{i}\right)=\sum_{k=1}^{N_{p}} v_{k}^{p}\left(t_{i}\right)+b,
$$

where $b$ is the RV offset and independent offsets are adopted for different instruments. The RV variation caused by planet $k$ is

$$
v_{k}^{p}\left(t_{i}\right)=\sqrt{\frac{G m_{p k}^{2}}{\left(m_{p k}+m_{s}\right) a_{k}\left(1-e_{k}^{2}\right)}} \sin I_{k}\left[\cos \omega_{k}+v_{k}\left(t_{i}\right)+e_{k} \cos \left(\omega_{k}\right)\right],
$$

where $m_{p k}$ is the mass, $a_{k}, e_{k}, I_{k}, \omega_{k}$ and $v_{k}$ are the orbital elements of the $k^{\text {th }}$ planet. The true anomaly and eccentricity anomaly are derived from the mean anomaly at the reference epoch for a given time. Independent sets of parameters of the MA model are applied to different data sets. We use a superscript to denote the parameter for a given set. For example, $\tau^{\text {HARPSpre }}$ is the correlation time scale for the HARPSpre data set.

\section{CONFIRMATION OF A COLD JUPITER}

A significant RV trend has been found in the CES and HARPS data sets (Endl et al. 2002, Zechmeister et al.|2013), suggesting a long-period planetary companion to $\epsilon$ Indi A. To constrain this RV trend better by including newer HARPS and UVES data, we analyze the CES LC and VLC data sets in combination with the HARPSpre, HARPSpost, and UVES sets. The LC and VLC data sets are corrected by accounting for the $1.8 \mathrm{~m} \mathrm{~s}^{-1} / \mathrm{yr}$ acceleration caused by the proper motion (so-called "perspective acceleration"). Based on Bayesian model comparison (Feng, Tuomi \& Jones 2017a), we model the trend in the combined data using one Keplerian function, and model the noise in LC, VLC, and UVES using white noise. We use the first and second order moving average models (i.e. MA(1) and MA(2)) respectively to model the noise in HARPSpre and HARPSpost, in the same manner as e.g., Tuomi et al. (2013); Feng et al. (2016).

We also vary the offsets between data sets and adopt the prior distributions for all parameters from Feng et al. (2016). Specifically, we use a one-sided Gaussian prior distribution $\mathcal{N}(0,0.2)$ for eccentricity to account for the eccentricity distributions found in radial velocity planets (Kipping 2013) and in transit systems (Kane et al. 2012, Van Eylen et al. 2019). Since there is no universal eccentricity distribution for all types of planets and stars, our use of a semi-Gaussian only captures the broad feature of the real eccentricity distributions. Nevertheless, we will test the sensitivity of our results to eccentricity priors later. We use a log uniform distribution for time scale parameters, and a uniform distribution for other parameters. Based on adapted MCMC samplings (Haario et al. 2006), we find a significant signal at a period of $17155_{-474}^{+6940} \mathrm{~d}$ or $47_{-13}^{+19} \mathrm{yr}$, which is well constrained by MCMC samplings. The one-planet model improves the maximum likelihood by about $10^{144}$ (or $\mathrm{BF}=10^{136}$ ) compared with the baseline model. It increases the maximum likelihood by about $10^{37}$ (or $\mathrm{BF}=10^{31}$ ) compared with the baseline model combined with a linear trend, and increase the maximum likelihood by $10^{22}$ (or $\mathrm{BF}=10^{18}$ ) compared with the baseline combined with a parabola. Hence a linear trend or parabola or a longer period signal is not favored by the data.

Since this trend is not found in noise proxies, it is likely to be caused by a wide-orbit planet with a minimum mass $m \sin I=2.6_{-0.72}^{+2.0} M_{\mathrm{Jup}}$, semi-major axis $a=12_{-2.0}^{+2.1} \mathrm{au}$, semi-amplitude $K=25_{-5.5}^{+16} \mathrm{~m} / \mathrm{s}$, eccentricity of $e=0.26_{-0.071}^{+0.078}$, argument of periapsis $\omega=60_{-16}^{+35} \mathrm{deg}$, and mean anomaly of $M_{0}=170_{-82}^{+50} \mathrm{deg}$ at the first epoch (or BJD2448929.56) of the combined 


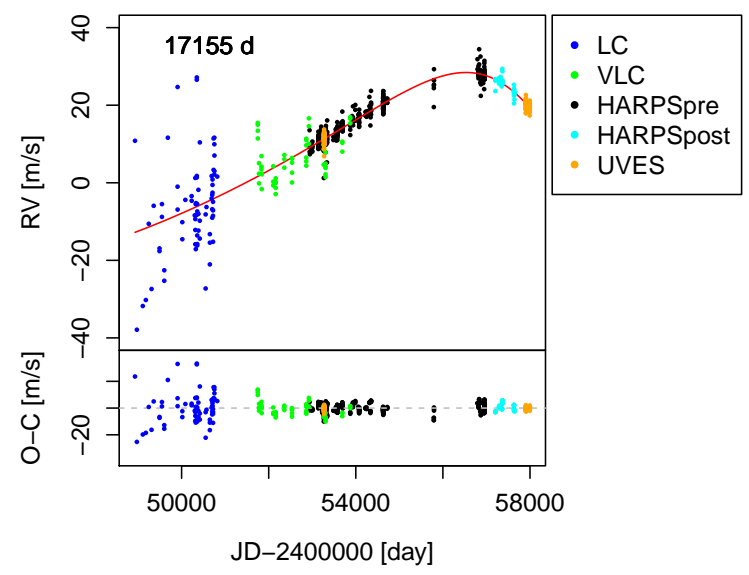

Figure 2. Best-fit for the data sets of CES-LC, CES-VLC, HARPSpre, HARPSpost, and UVES. The offset and correlated noise are subtracted from the data. The red line denotes the Keplerian signal with a period of $47 \mathrm{yr}$ based on the analysis of RV data.

RV data. The optimal value is determined at the maximum a posteriori and the uncertainties correspond to the 10 and $90 \%$ quantiles of the posterior distributions. This solution for $\epsilon$ Indi Ab is shown in Fig. 2, The offset between VLC and LC sets is $4.6 \pm 2.8 \mathrm{~m} / \mathrm{s}$, which is within the uncertainty of $8 \mathrm{~m} / \mathrm{s}$ determined from a sample of VLC and LC sets (Zechmeister et al. 2013). The offset between the HARPSpre and HARPSpost set is $12 \pm 1.3 \mathrm{~m} / \mathrm{s}$, consistent with the offset between pre-and-post fibre exchange for a K2 star given by Lo Curto et al. (2015). The mass determined in this work is consistent with the range given in Janson et al. (2009), who adopt a $2.6 \mathrm{~m} \mathrm{~s}^{-1} /$ yr planet-induced acceleration of $\epsilon$ Indi A relative to its barycenter (or stellar reflex motion) determined from epochs earlier than JD2455000 (or June, 2009), leading to an estimation of mass limit of $5-20 M_{\mathrm{Jup}}$. On the other hand, Zechmeister et al. (2013) adopt a $2.4 \mathrm{~m} \mathrm{~s}^{-1} / \mathrm{yr}$ slope and find a broad limit of $M$ sin $i>0.97 M_{\mathrm{Jup}}$ and $P>30$ yr. With the benefit of the number of years of precise HARPS epochs we are in a position to determine a more modest slope value of $1.8 \mathrm{~m} \mathrm{~s}^{-1} \mathrm{yr}^{-1}$ for HARPSpre epochs and a negative slope value of $-2.9 \mathrm{~m} \mathrm{~s}^{-1} \mathrm{yr}^{-1}$ for the HARPSpost epochs. Moreover, the combination of UVES and HARPS sets also strongly favor a curvature in the RV variation. The early epochs of UVES overlap with HARPS epochs and thus enable a good offset calibration. The recent epochs of UVES suggest a significant decrease in RV (see Fig. 2) as already seen in the HARPSpost set. Thus a Keplerian function is more appropriate than the previously used linear trend to model the RV variation and constrain the signal.

Since $\epsilon$ Indi $\mathrm{Ab}$ is on such a very wide orbit and so within the dataset of known planets can not be expected to follow the eccentricity distribution found in relatively short period planets (Kipping 2013; Jurić \& Tremaine 2008). Hence we test whether the values of orbital parameters are sensitive to the choice of eccentricity prior. We change the standard deviation of the one-sided Gaussian prior from 0.2 to 0.4 , and also adopt a uniform eccentricity prior. The orbital parameters for different parameters and the $\log$ BF are shown in Table 1. It is evident that the orbital parameters from different solutions and the offsets as well as jitters are consistent with each other and are thus not sensitive to the choice of eccentricity prior.

\section{DIAGNOSTICS OF SIGNALS USING BAYES FACTOR PERIODOGRAMS}

In this section we focus on the constraints provided by the long-term HARPS dataset alone and investigate evidence for other signals within the data sets of HARPS, HARPSlow, and HARPSpre using the Agatha software (Feng, Tuomi \& Jones 2017a), which is essentially a framework of red noise periodograms. By comparison of different noise models in Agatha, we find that a variety of optimal noise models: (1) HARPS - fifth order moving average (or MA(5)) in combination with FWHM and NaD1, (2) HARPSlow - MA(2) combined with BIS and NaD1, (3) HARPSpre - MA(2) combined with S-index and NaD1.

To find primary signals, we calculate the Bayes factor periodogram (BFP) for the MA(1) model in combination with proxies for different data sets, and show them in Fig. 3 . In this figure, the signal around $11 \mathrm{~d}$ is significant in the HARPS data set because the high cadence data favors short period signals. There are also strong powers around this signal for the HARPSlow and HARPSpre data sets even though the high cadence and HARPSpost epochs have been excluded. For all data sets, the signal at $18 \mathrm{~d}$ is significant although the HARPS data set favors the $11 \mathrm{~d}$ signal more. The rather strong and noisy power around $11 \mathrm{~d}$ in the left panels are contributed by 11 days of consecutive data which comprise the high cadence RVs.

In particular, the signal at a period of $278 \mathrm{~d}$ is significant in the BFPs for MA(1) but become much weaker in the BFPs for MA(1) combined with noise proxies, suggesting an activity origin. The other strong signals in these BFPs are either aliases or harmonics of these three signals, as we will see in the subsequent analysis. 
Table 1. Sensitivity of orbital parameters to the choice of eccentricity priors. The optimal value is calculated at the maximum a posteriori (MAP) and the uncertainties correspond to the $10 \%$ and $90 \%$ qantiles. The $\ln (\mathrm{BF})$ of one-planet model with respect to the baseline model is estimated by the Bayesian information criterion (BIC; Schwarz et al. 1978), and $\ln (\mathrm{BF})>5$ is the signal detection threshold (Feng, Tuomi \& Jones 2017a).

\begin{tabular}{|c|c|c|c|}
\hline & $P(e)=2 \mathcal{N}(0,0.2)$ & $P(e)=2 \mathcal{N}(0,0.4)$ & $\begin{array}{c}P(e)=1 \\
0<e<1\end{array}$ \\
\hline$P[\mathrm{yr}]$ & $47_{-13}^{+19}$ & $44_{-9}^{+23}$ & $49_{-14}^{+19}$ \\
\hline$K[\mathrm{~m} / \mathrm{s}]$ & $25_{-5}^{+16}$ & $29_{-9}^{+13}$ & $28_{-7.9}^{+1.7}$ \\
\hline$e$ & $0.26_{-0.077}^{+0.078}$ & $0.26_{-0.049}^{+0.11}$ & $0.26_{-0.054}^{+0.11}$ \\
\hline$\omega[\mathrm{deg}]$ & $60_{-16}^{+35}$ & $80_{-36}^{+17}$ & $64_{-18}^{+32}$ \\
\hline $\boldsymbol{M}_{0}[\mathrm{deg}]$ & $170_{-82}^{+50}$ & $140_{-54}^{+80}$ & $167_{-80}^{+51}$ \\
\hline$b^{\mathrm{LC}}$ & $-17_{-15}^{+7.7}$ & $-17_{-16}^{+7.7}$ & $-19_{-15}^{+9.7}$ \\
\hline$\sigma^{\mathrm{LC}}$ & $4.2_{-2.2}^{+2.9}$ & $3.5_{-1.5}^{+3.5}$ & $2.8_{-0.79}^{+4.2}$ \\
\hline$b^{\mathrm{VLC}}$ & $-20_{-17}^{+6.9}$ & $-22_{-15}^{+8.8}$ & $-23_{-17}^{+9.7}$ \\
\hline$\sigma^{\mathrm{VLC}}$ & $0.14_{--0.13}^{+2.4}$ & $0.59_{-0.35}^{+1.9}$ & $0.52_{-0.25}^{+2.1}$ \\
\hline$b^{\text {HARPSpre }}$ & $-25_{-17}^{+7.5}$ & $-27_{-15}^{+9}$ & $-29_{-17}^{+9.9}$ \\
\hline$\sigma^{\text {HARPSpre }}$ & $1.5_{-0}^{+0.055}$ & $1.4_{-0.024}^{+0.11}$ & $1.4_{-0.034}^{+0.099}$ \\
\hline$w_{1}^{\text {HARPSpre }}$ & $0.67_{-0.027}^{+0.09}$ & $0.68_{-0.038}^{+0.081}$ & $0.68_{-0.038}^{+0.078}$ \\
\hline$w_{2}^{1 \text { HARPSpre }}$ & $0.34_{-0.10}^{+0.028}$ & $0.31_{-0.066}^{+0.036}$ & $0.35_{-0.11}^{+0.0 .028}$ \\
\hline $\ln \frac{\tau^{\text {HARPSpre }}}{1 \text { day }}$ & $0.97_{-0.18}^{+0.26}$ & $1.1_{-0.34}^{+0.082}$ & $1.0_{-0.21}^{+0.21}$ \\
\hline$b^{\text {HARPSpost }}$ & $-12_{-18}^{+7.2}$ & $-17_{-13}^{+11}$ & $-15_{-18}^{+9.5}$ \\
\hline$\sigma^{\text {HARPSpost }}$ & $1.0_{-0.083}^{+0.21}$ & $1.0_{-0.056}^{+0.24}$ & $1.1_{-019}^{+0.11}$ \\
\hline$w_{1}^{\text {HARPSpost }}$ & $0.92_{-0.051}^{-0.083}$ & $\begin{array}{c}-0.056 \\
0.97_{-0.097}^{+0.016}\end{array}$ & $0.92_{-0.053}^{-0.19}$ \\
\hline $\ln \frac{\tau^{\text {HARPSpost }}}{1 \text { day }}$ & $2.6_{-0.77}^{+3.951}$ & $6_{-4.2}^{+0.27}$ & $\begin{array}{l}2.7_{-0.9}^{+3.7} \\
\end{array}$ \\
\hline$b^{\mathrm{UVES}}$ & $-12_{-17}^{+7.6}$ & $-15_{-15}^{+9}$ & $-16_{-17}^{+9.7}$ \\
\hline$\sigma^{\mathrm{UVES}}$ & $0.63_{-0.14}^{+0.085}$ & $0.6_{-0.11}^{+0.12}$ & $0.58_{-0.086}^{+0.15}$ \\
\hline $\ln (\mathrm{BF})$ & 314 & 314 & 314 \\
\hline
\end{tabular}

Since the HARPSpre data set is more conservative than the HARPShigh and HARPSlow sets due to its sampling and is not subject to the uncertainty in radial velocity offset due to fibre change, we calculate the BFPs for the raw HARPSpre data and the RV residual signals and for various noise proxies, and show these BFPs in Fig. 4 In panels P1-P14, we observed that the signals at periods of about 11,18 , and $278 \mathrm{~d}$ are significant in the data. The $278 \mathrm{~d}$ signal is likely caused by activity because its significance is reduced after accounting for the correlation between RVs and noise proxies, as seen in P5-P7. This signal is found to be significant in the BFPs of the $\mathrm{NaD} 1, R_{\mathrm{HK}}, \mathrm{NaD} 2$, and $\mathrm{H} \alpha$ indices. In addition, the signal at a period of around $2500 \mathrm{~d}$ is also significant in the BFPs of NaD1 (P9), $\mathrm{R}_{H K}^{\prime}(\mathrm{P} 13), \mathrm{NaD} 2(\mathrm{P} 17)$, and BIS (P23 and P24). We interpret this signal as the magnetic cycle and the $278 \mathrm{~d}$ signal as a secondary cycle in the magnetic variation. We also observe strong signals around $18 \mathrm{~d}$ and its double period $36 \mathrm{~d}$ in $\mathrm{NaD} 1, \mathrm{NaD} 2, \mathrm{R}_{H K}^{\prime}$, and BIS. Nevertheless, the $18 \mathrm{~d}$ signal does not disappear in the BFPs accounting for linear correlations between RVs and NaD1 and $\mathrm{R}_{H K}^{\prime}$. Hence we conclude that this signal is either due to a nonlinear effect of stellar rotation or due to a planet, which has an orbital period similar to rotation period. The signal at about $11 \mathrm{~d}$ is found to be unique and significant in the residual of NaD1 after subtraction of the 2500 and $18 \mathrm{~d}$ signals. Thus this signal is probably caused by stellar activity or is an alias of activity-induced signals. We also look into the ASAS and Hipparcos photometric data but find few useful epochs and identify no significant signals.

Therefore we conclude that the primary and secondary magnetic cycles of $\epsilon$ Indi are 2500 and $278 \mathrm{~d}$. The rotation period of $\epsilon$ Indi is about $36 \mathrm{~d}$, approximately double $18 \mathrm{~d}$. This rotation period is rather different from the $22 \mathrm{~d}$ value estimated by Saar \& Osten (1997) from Ca II measurements. Considering that the $36 \mathrm{~d}$ rotation period is derived from a relatively large dataset of high precision RVs and multiple activity indicators, we believe that $36 \mathrm{~d}$ is a more reliable value of rotation period. On the other hand, the half rotation period, $18 \mathrm{~d}$, is more significant than the rotation period in the RV data. This phenomenon is also found in the RVs of other stars (e.g. HD 147379; Feng, Jones \& Tuomi 2018), and is probably caused by a spot or spot complexity which more significantly modulates spectral lines over one half of the rotation period. The signal at a period of about $11 \mathrm{~d}$ is also related to the stellar rotation since $1 /(1 / 36+1 / 18) \approx 11$. Based on the Bayesian quantification of these signals using the MA(1) model (Tuomi et al. 2013, Feng et al. 2016), the semi-amplitudes of the signals at periods 


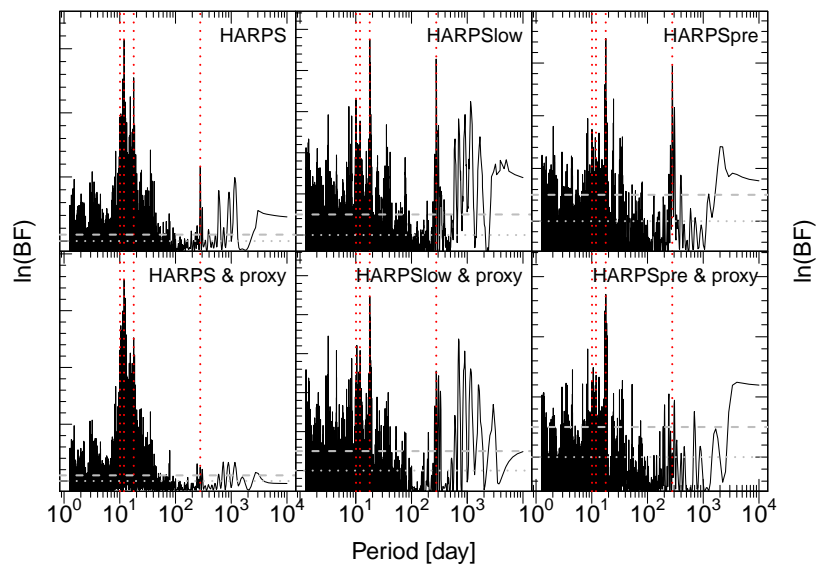

Figure 3. BFPs for MA(1) model in combination with noise proxies for the HARPS (left), HARPSlow (middle) and HARPSpre (right) data sets. The upper panels show the BFPs for the MA(1) model while the lowers ones for the MA(1) model in combination with the optimal noise proxies. The signals at periods of $10,12,18$ and $278 \mathrm{~d}$ are shown by dotted red lines. The thresholds of $\ln (\mathrm{BF})=0$ and 5 are shown by the horizontal dotted and dashed lines, respectively.

of $10.064_{-0.001}^{+0.003} 17.866_{-0.003}^{+0.006}$ and $278_{-0.60}^{+1.7} \mathrm{~d}$ signals are $1.4_{-0.31}^{+0.18}, 2.1_{-0.19}^{+0.37}$, and $2.3_{-0.31}^{+0.34} \mathrm{~m} / \mathrm{s}$, respectively. The non-detection of additional signals puts an upper limit of $1 \mathrm{~m} / \mathrm{s}$ on the semi-amplitude RV variation induced by potential planets with periods less than the HARPSpre baseline (i.e. $P<4000$ days) in the system.

Based on the above analysis, different noise proxies are sensitive to different stellar activities. NaD1 and $\mathrm{R}_{H K}^{\prime}$ are sensitive to all activity-induced signals while BIS is sensitive to stellar rotation signal. $\mathrm{H} \alpha$ is only sensitive to the secondary magnetic cycle. Considering the differential sensitivity of activity indicators to activity signals, a model including a linear correlation between RVs and these indicators would remove activity-noise as well as introduce extra noise. This is evident from the comparison of P1 with P5 in Fig. 4. Although the $278 \mathrm{~d}$ signal disappears after accounting for the linear correlation with S-index and NaD1, a longer period around $3000 \mathrm{~d}$ and some short-period signals become significant (also compare P2 and P6, P3 and P7). Such extra signals are caused by fitting an inappropriate linear function of noise proxies to RVs while these proxies are not perfectly correlated with RVs linearly. Hence these proxies would bring their own signals into the likelihood/posterior distribution, which is a combined function of data and model, in order to mitigate the main activity signals in RVs. While the traditional activity indicators are limited in removing activity-induced signals, differential RVs are better in removing wavelength-dependent signals without introducing extra noise because they provide a simple way to weight spectral orders $a$ posteriori (Feng et al. 2017). Hence a reliable diagnostics of the nature of signals is to test the sensitivity of signals to different noise models, data chunks, and wavelengths.

Since the activity-induced RV variations are not strictly periodic, Keplerian functions or a Gaussian process model cannot adequately model them. Hence the fit of a one-planet model is appropriate for parameter inference (see section 3 ).

\section{COMBINED ANALYSIS OF RV AND ASTROMETRY}

\subsection{Combined model of RV and astrometry}

In a star-planet or more generally star-companion system, the position of the star in its orbital plane is calculated according to KeplerâĂŹs equation is

$$
\boldsymbol{r}_{S}(t)=\left[\begin{array}{c}
x(t) \\
y(t) \\
0
\end{array}\right]=\frac{m_{p}}{m_{p}+m_{s}} a\left[\begin{array}{c}
\cos E(t)-e \\
\sqrt{1-e^{2}} \sin E(t) \\
0
\end{array}\right],
$$

where $m_{s}$ and $m_{p}$ are respectively the masses of star and planet, $a$ is the semi-major axis of the planet with respect to the star, $E(t)$ is the eccentricity anomaly, and $e$ is the eccentricity. The corresponding stellar reflex velocity is

$$
\boldsymbol{v}_{S}(t)=\left[\begin{array}{c}
v_{x}(t) \\
v_{y}(t) \\
0
\end{array}\right]=\frac{G m_{p}^{2}}{\left(m_{p}+m_{S}\right) a\left(1-e^{2}\right)}\left[\begin{array}{c}
-\sin v(t) \\
\cos v(t)+e \\
0
\end{array}\right],
$$

\footnotetext{
2 Since the 10 and 12 day signals are aliases of each other, we only report one signal here.
} 


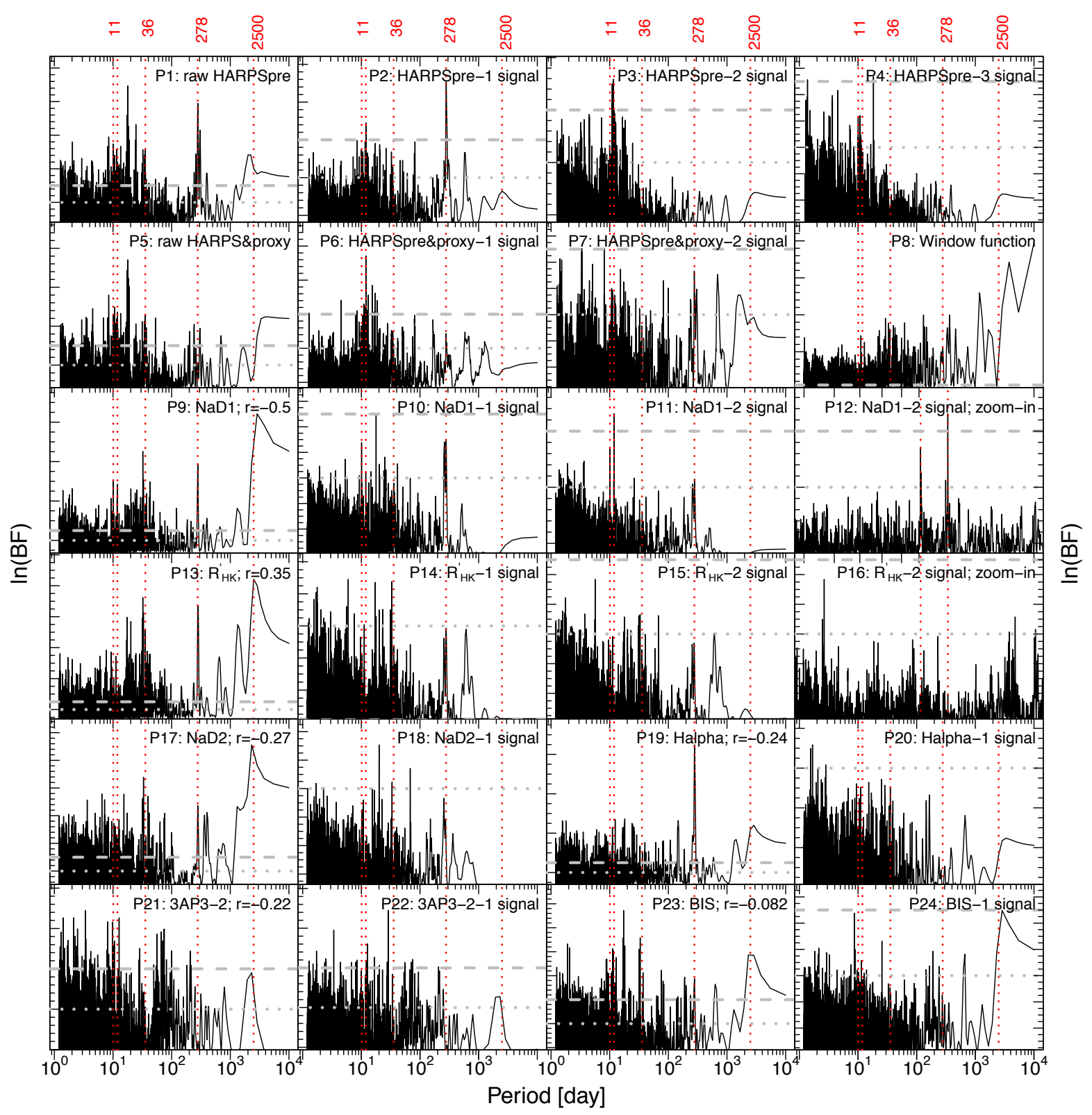

Figure 4. BFPs for RVs and noise proxies. Top row: BFPs for MA(1) for raw HARPSpre and HARPSpre subsequently subtracted by signals at periods of $11,18,36,278$, and $2500 \mathrm{~d}$. The $11 \mathrm{~d}$ signal is denoted by two periods at 10 and $12 \mathrm{~d}$ according to posterior samplings. These periods are shown in the top of the figure. Second row: BFPs for MA(1) combined with S-index and NaD1 for raw HARPSpre and HARPSpre subtracted in turn by signals at periods at 18 and $11 \mathrm{~d}$, and the Lomb-Scargle periodogram for the window function (P8). The third row onward shows BFPs for MA(1) for noise proxies and their residuals. P12 and P16 are respectively the zoom-in versions of P11 and P15 and have a period range of $[5,20]$ d. For each panel, the Pearson correlation coefficient in the top right corner shows the correlation between noise proxies and RVs which are detrended by a second order polynomial. All panels are denoted by "Pn" with $\mathrm{n}$ varying from 1 to 24 . The horizontal dotted and dashed lines denote $\ln \mathrm{BF}=0$ and 5 , respectively. The BFP for the FWHM is not shown because there is not significant periodicity. The values of $\ln (\mathrm{BF})$ are indicated by thresholds 0 and 5 rather than shown by axis labels since different panels have different ranges of $\ln (\mathrm{BF})$.

where $v(t)$ is the true anomaly at time $t, G$ is the gravitational constant. The stellar position $\boldsymbol{r}_{s}(t)$ is converted to the observer frame coordinates, $\boldsymbol{r}_{s}^{\mathrm{obs}}(t)$, by applying Euler rotations using

$$
r_{s}^{\mathrm{obs}}=R_{Z}(\Omega) R_{X}(-I) R_{Z}(\omega) \boldsymbol{r}_{s}(t),
$$

where $\Omega$ is the longitude of ascending node, $\omega$ is the argument of periastron, $I$ is the inclination of the stellar orbit with respect to the sky plane. The directions of X axis (along North of the sky plane), Y axis (along East of the sky plane), and 
$\mathrm{Z}$ axis (along the line of sight towards the observer) forms a right-handed Cartesian coordinate system in the observer frame. The expansion of equation (5) gives the observed location of star in the XYZ coordinate system,

$$
\left[\begin{array}{c}
x^{\mathrm{obs}}(t) \\
y^{\mathrm{obs}}(t) \\
z^{\mathrm{obs}}(t)
\end{array}\right]=\left[\begin{array}{ccc}
A & F & -\sin \Omega \sin I \\
B & G & \cos \Omega \sin I \\
-\sin \omega \sin I & -\cos \omega \sin I & \cos I
\end{array}\right]\left[\begin{array}{c}
x(t) \\
y(t) \\
0
\end{array}\right],
$$

where

$$
\begin{aligned}
& A=\cos \Omega \cos \omega-\sin \Omega \sin \omega \cos I \\
& B=\sin \Omega \cos \omega+\cos \Omega \sin \omega \cos I \\
& F=-\cos \Omega \sin \omega-\sin \Omega \cos \omega \cos I \\
& G=-\sin \Omega \sin \omega+\cos \Omega \cos \omega \cos I
\end{aligned}
$$

are the so-called Thiele Innes constants. Hence the variation of right ascension, declination, and parallax caused by a planet at a given time is

$$
\begin{aligned}
& \alpha^{p}(t)=\tilde{\omega}(t) y^{\mathrm{obs}}(t)=\tilde{\omega}(t)[B x(t)+G y(t)], \\
& \delta^{p}(t)=\tilde{\omega}(t) x^{\mathrm{obs}}(t)=\tilde{\omega}(t)[A x(t)+F y(t)],
\end{aligned}
$$

The stellar reflex velocity is the time derivative of $\boldsymbol{r}_{s}^{\mathrm{obs}}(t)$, which is

$$
\begin{aligned}
& \mu_{\alpha}(t)=\tilde{\omega}(t)\left[B v_{x}(t)+G v_{y}(t)\right], \\
& \mu_{\delta}^{p}(t)=\tilde{\omega}(t)\left[A v_{x}(t)+F v_{y}(t)\right],
\end{aligned}
$$

The planet-induced RV is

$$
v_{r}^{p}=-v_{z}^{\mathrm{obs}}=v \sin I[\cos (\omega+v)+e \cos \omega] .
$$

The stellar motion is the sum of the barycentric motion (denoted by superscript $b$ ) and the reflex motion (denoted by superscript $p$ ) described by

$$
\begin{aligned}
\alpha(t) & =\frac{\alpha^{p}(t)}{\cos \delta(t)}+\alpha^{b}(t), \\
\delta(t) & =\delta^{p}(t)+\delta^{b}(t), \\
\frac{1}{\tilde{\omega}} & =-z^{\mathrm{obs}}(t)+\frac{1}{\tilde{\omega}^{b}(t)}, \\
\mu_{\alpha}(t) & =\mu_{\alpha}^{p}(t)+\mu_{\alpha}^{b}(t), \\
\mu_{\delta}(t) & =\mu_{\delta}^{p}(t)+\mu_{\delta}^{b}(t), \\
v_{r}(t) & =v_{r}^{p}(t)+v_{r}^{b}(t) .
\end{aligned}
$$

Note that the distance or the inverse of parallax rather than the parallax is additive. $\alpha^{p}(t)$ is a planet-induced offset in the sky plane and thus should be divided by $\cos \delta(t)$ to calculate the real change in righ ascension. Assuming zero acceleration, the heliocentric motion of the barycenter of a planetary system is determined by

$$
\begin{aligned}
& \boldsymbol{v}^{b}(t)=\boldsymbol{v}^{b}\left(t_{0}\right) \\
& \boldsymbol{r}^{b}(t)=\boldsymbol{r}^{b}\left(t_{0}\right)+\boldsymbol{v}^{b}\left(t_{0}\right)\left(t-t_{0}\right)
\end{aligned}
$$

where $\boldsymbol{v}^{b}(t)$ is the barycentric velocity, and $\boldsymbol{r}^{b}(t)$ is the barycentric position. The linear motion is a good approximation of heliocentric motion of the barycenter over a few decades since the Galactic acceleration for nearby stars is typically less than $\mathrm{mm} / \mathrm{s} /$ year.

Since the RV variation induced by the barycentric motion, $v_{r}^{b}(t)$, is subtracted from the RV data, we only use $v_{r}^{p}(t)$ defined in equation (9) to model the Keplerian signal in RV data. Considering the barycentric RV is used to calculate the astrometry variation caused by the change of perspective, the planet-induced RV variation or gravitational redshift and convection blueshift only contribute as a secondary variation in astrometric data. Thus we approximate the barycentric RV by the Gaia RV if available or the value from the RV data sets we collected, i.e. $v_{r}^{b}(t) \approx v_{r}(t)$. We also neglect the parallax variation induced by stellar reflex motion and by the RV because they are secondary effects compared with the variation of other observables. For example, the parallax of $\epsilon$ Indi A is only changed by about $0.3 \mu$ as due to the barycentric motion along the line of sight. Thus we only model the astrometry observables $\alpha, \delta, \mu_{\alpha}$ and $\mu_{\delta}$ of Hipparcos and Gaia DR2 to constrain the planetary orbit. The procedure of astrometry modeling is as follows. 
- Choose the reference time at the Gaia epoch J2015.5 and determine the initial barycentric astrometry according to

$$
\begin{aligned}
\alpha^{b}\left(t_{0}\right) & =\alpha^{\text {gaia }}\left(t_{0}\right)-\frac{\alpha^{p}\left(t_{0}\right)}{\cos \delta^{\text {gaia }}\left(t_{0}\right)}-\Delta \alpha, \\
\delta^{b}\left(t_{0}\right) & =\delta^{\text {gaia }}\left(t_{0}\right)-\delta^{p}\left(t_{0}\right)-\Delta \delta, \\
\tilde{\omega}\left(t_{0}\right) & =\tilde{\omega}\left(t_{0}\right), \\
\mu_{\alpha}^{b}\left(t_{0}\right) & =\mu_{\alpha}^{\text {gaia }}\left(t_{0}\right)-\mu_{\alpha}^{\mathrm{p}}\left(t_{0}\right)-\Delta \mu_{\alpha}, \\
\mu_{\delta}^{b}\left(t_{0}\right) & =\mu_{\delta}^{\text {gaia }}\left(t_{0}\right)-\mu_{\delta}^{\mathrm{p}}\left(t_{0}\right)-\Delta \mu_{\delta}, \\
v_{r}^{b}\left(t_{0}\right) & =v_{r}\left(t_{0}\right),
\end{aligned}
$$

where $\Delta \alpha, \Delta \delta, \Delta \mu_{\alpha}$, and $\Delta \mu_{\delta}$ are the offsets. These offsets are used to account for the reference frame spin rate (about $0.15 \mathrm{mas} / \mathrm{yr}$ according to Lindegren et al. (2018)), for the zero point offset of parallaxes (about -0.029 mas according to Lindegren et al. (2018)), and for the proper motion offsets related to light travel time (about 0.6 mas/yr in the case of $\epsilon$ Indi A; Kervella et al. 2019), and for other effects.

- Transform initial astrometry and RV into heliocentric state vector $\left[\boldsymbol{r}^{b}\left(t_{0}\right), \boldsymbol{v}^{b}\left(t_{0}\right)\right]$, and propagate the vector from $t_{0}$ to $t$ to derive $\left[\boldsymbol{r}^{b}(t), \boldsymbol{v}^{b}(t)\right]$ according to equation 16 and transform the state vector back to barycentric astrometry at time t. This step is to calculate perspective acceleration.

- Estimate $\alpha(t), \delta(t), \mu_{\alpha}(t)$, and $\mu_{\delta}(t)$ by summing the barycentric and reflex astrometry according to equations 12,13 and 15.

To account for unknown noise in astrometry data, we include a relative jitter in units of observational errors in the likelihood. Thus the likelihood of the combined RV and astrometry model is

$$
\begin{aligned}
\ln \mathcal{L} & \equiv P(D \mid \theta, M)=\prod_{k}^{N_{\mathrm{set}}} \prod_{i}^{N_{k}^{\mathrm{rv}}} \frac{1}{\sqrt{2 \pi\left(\sigma_{j}^{2}+\sigma_{\mathrm{Jk}}^{2}\right)}} \exp \left\{-\frac{\left[\hat{v}_{r}\left(t_{i}\right)-v_{r}\left(t_{i}\right)^{2}\right]}{2\left(\sigma_{i}^{2}+\sigma_{\mathrm{Jk}}^{2}\right)}\right\} \\
& +(2 \pi)^{\frac{N_{\mathrm{epoch}} N_{\mathrm{par}}}{2}} \prod_{j}^{N_{\text {epoch }}}\left(\operatorname{det} \Sigma_{j}\right)^{-\frac{1}{2}} \exp \left\{-\frac{1}{2}\left[\hat{\boldsymbol{\eta}}\left(t_{i}\right)-\boldsymbol{\eta}\left(t_{i}\right)\right]^{T} \Sigma_{j}^{-1}\left[\hat{\boldsymbol{\eta}}\left(t_{i}\right)-\boldsymbol{\eta}\left(t_{i}\right)\right]\right\},
\end{aligned}
$$

where $N_{\text {set }}, N_{\text {epoch }}$, and $N_{\text {par }}$ are respectively the number of RV data sets, astrometry epochs, and free parameters of the astrometry model. $N_{k}^{\mathrm{rv}}$ is the number of RVs in the $k^{\text {th }} \mathrm{RV}$ set, $\boldsymbol{\eta} \equiv\left[\alpha, \delta, \mu_{\alpha}, \mu_{\delta}\right]$ is the astrometry data, and $\Sigma$ is the jitter corrected covariance matrix of $\boldsymbol{\eta}, \Sigma_{j} \equiv \Sigma_{0 \mathrm{j}}\left(1+J_{j}\right)$ where $\Sigma_{0 \mathrm{j}}$ is the catalog covariance matrix for the $j^{\text {th }}$ astrometry epoch, and $J_{j}$ is the so-called "relative astrometry jitter". These relative jitters allow the model to account for potential underestimation of astrometry errors such as the so-called DOF bug (Lindegren et al. 2018) and the effects mentioned in Brandt (2018). Unlike previous calibrations of the five-parameter solutions of Hipparcos and Gaia DR2 based on statistical analyses (Lindegren et al. 2018, Brandt 2018), we model the potential underestimation of uncertainties and bias as offsets and astrometric jitters, and estimate them a posteriori rather than a priori by taking advantage of the high precision RV data and a combined modeling of the barycentric and reflex motions of $\epsilon$ Indi A. The RV model $\hat{v}_{r}\left(t_{i}\right)$ is given in Eqn. 1

Therefore the free parameters in the combined model are $\left\{m_{p k}, a_{k}, e_{k}, I_{k}, \omega_{k}, \Omega_{k}, M_{0 k}\right\}$ with $k \in\left\{1, \ldots, N_{p}\right\}$ for $N_{p}$ planets or companions, $\left\{\Delta \alpha, \Delta \delta, \Delta \mu_{\alpha}, \Delta \mu_{\delta}, J_{\text {gaia }}, J_{\text {hip }}\right\}$ for astrometry, $\left\{b_{1}, \ldots, b_{N_{\text {set }}}\right\}$ for the offsets and $\sigma_{1}, \ldots, \sigma_{N_{\text {set }}}$ for the jitters in $N_{\text {set }}$ independent RV sets, $\left\{w_{1}^{i}, \ldots, w_{q}^{i}\right\}$ and $\tau^{i}$ for the MA(q) model for the $i^{\text {th }}$ RV data set. The superscript of a given parameter denotes the name of the corresponding data set. The argument of perihelion and semi-major axis for a planetary orbit with respect to the barycenter are $\omega_{p}=\omega+\pi$ and $a_{p}=m_{s} /\left(m_{p}+m_{s}\right) a$ while the other angular parameters are the same as for the stellar reflex motion.

The offsets between Gaia and Hipparco catalog data are also used by Calissendorff \& Janson (2018); Brandt (2018); Kervella et al. (2019) to constrain the dynamic mass of massive companions and to identify potential companion-induced accelerations. In particular, Snellen \& Brown (2018) used both the proper motion difference and Hipparcos epoch data to constrain the mass of $\beta$ Pictoris b. While these studies justify use of astrometry difference between Hipparcos and Gaia DR2 to constrain stellar reflex motion, the constraints are not strong due to a lack of combined modeling of RV and the difference in proper motion as well as in position. In particular, we find that position difference is found to be more sensitive to non-linear stellar motions which appear from orbital integration over decades. The aim of this work is to focus on a target with clear-cut RV and astrometric evidence alongside appropriate treatment of Gaia and Hipparcos catalog data in combination with RV analysis.

\subsection{Orbital solution}

Based on the MCMC sampling of the posterior, we find the best orbital solution and show the fit to RV and astrometry data in Fig. 5. In panel (A), it is visually apparent that the one-planet model is strongly favored relative to the zero-planet model 
(A)

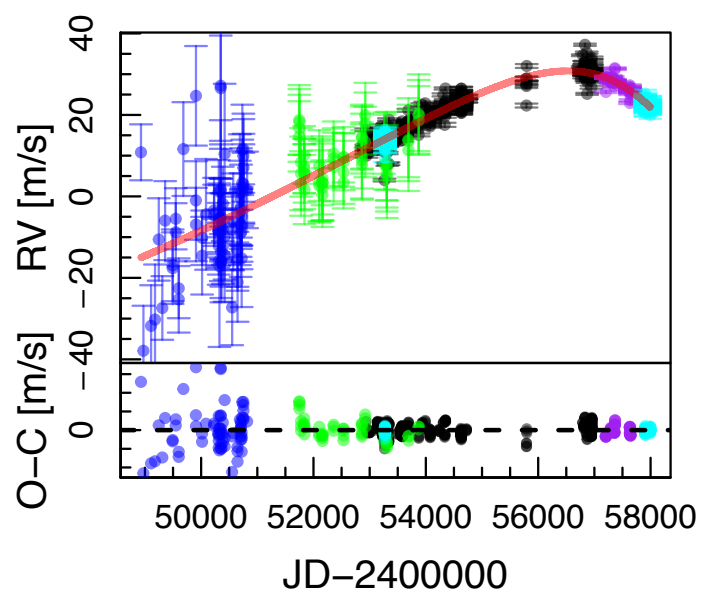

(B)

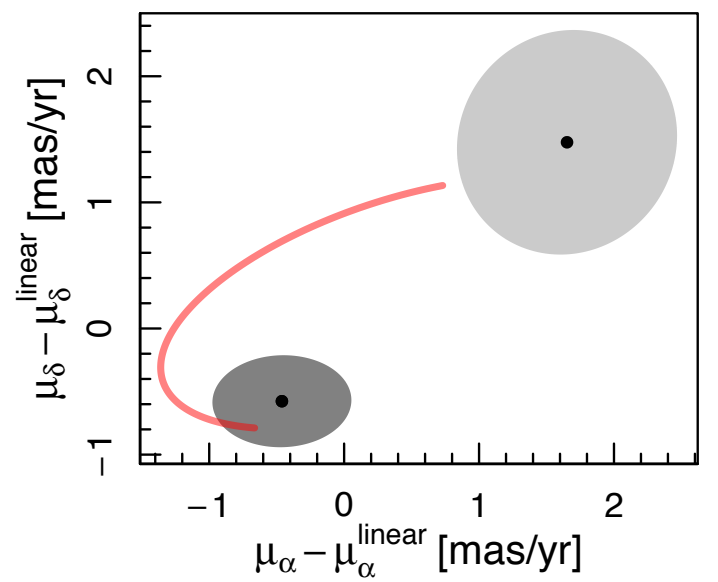

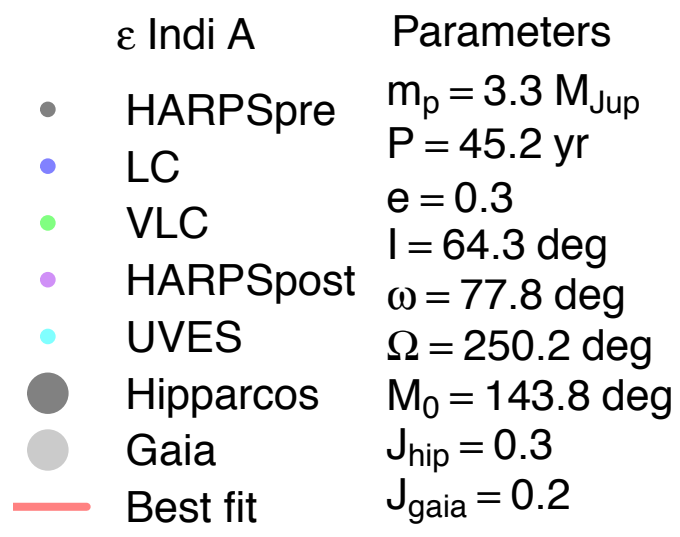

(C)

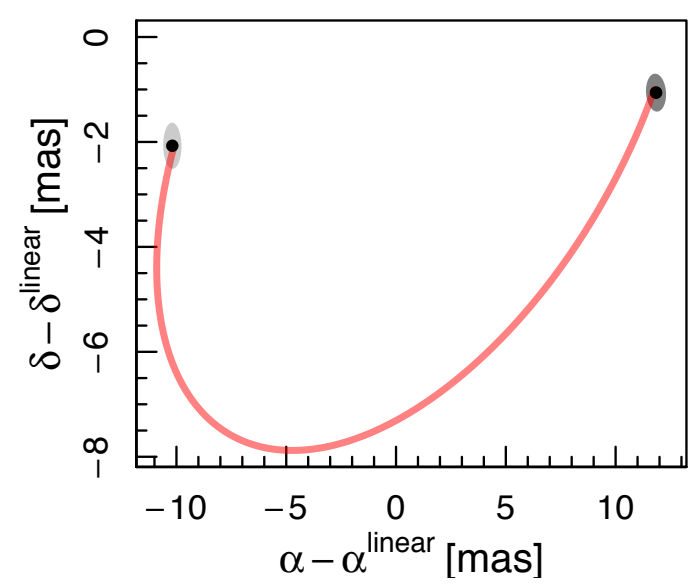

Figure 5. Observed RV data (A) and astrometric data in terms of proper motion (B) and position (C) compared to best fit model predictions. The offsets due to linear motion are subtracted from the data. (A) RV data of $\epsilon$ Indi A compared to the best fit model parameters (maximum a posteriori) represented as a red line with values shown in the legend. $\boldsymbol{J}_{\text {gaia }}$ and $\boldsymbol{J}_{\text {hip }}$ are respectively the relative excess noise in Gaia and Hipparcos astrometry data. (B) Hipparcos and Gaia DR2 proper motions are respectively denoted by the dark and light gray ellipse with a black dot in the center. The red line denotes the same "best" model prediction of the proper motion shown in (A). The ellipses show the 90\% jitter-corrected confidence level of astrometry. (C) The relative Hipparcos position at BJD2448349.0625 and Gaia DR2 position at BJD2457206.375 are shown along with the red line showing the best model prediction of the position. The barycentric motion of $\epsilon$ Indi A is subtracted from data in order to show its reflex motion.

$(\ln \mathcal{L}=331$ or $\ln \mathrm{BF}=311)$ and that the curvature in the $\mathrm{RV}$ data is significant compared with a linear trend $(\ln \mathcal{L}=51$ or $\ln \mathrm{BF}=38)$. Fig. 5 (B) and (C) indicate that the position puts a stronger constraint on the best fit model of the planetary orbit than the proper motion and comparable to the highest precision RV data. This arises because the position difference serves to integrate the non-linear proper motion over time whereas the proper motion difference is not as sensitive as position data to non-linear motion due to potential bias caused by the assumption of linear motion in the production of catalog data.

Based on the combined analysis, we report the values of the orbital parameters of $\epsilon$ Indi A b together with the stellar parameters in Table 2. Note that the stellar mass slightly differs from the value given by Demory et al. (2009) because we determine the mass using the Gaia luminosity and the mass-luminosity relationship derived by Eker et al. (2015). The posterior distribution of these parameters is shown in Fig. A1 In particular, we show the posterior distribution of planetary mass and orbital period in Fig. B1. It is apparent that the planetary mass and orbital period are constrained to a relatively high precision even without RV and astrometry data that cover the whole orbital phase.

Based on the above analysis, $\epsilon$ Indi $\mathrm{Ab}$ is a cold Jovian exoplanet with a very long orbital period among the exoplanets detected through the radial velocity and transit methods. With components $\mathrm{A}, \mathrm{Ab}, \mathrm{Ba}$ and $\mathrm{Bb}, \epsilon$ Indi provides a benchmark system for the formation of gas giants and brown dwarfs. $\epsilon$ Indi Ab is also a perfect target for direct imaging. The non-detection in previous direct imaging suggests a very low temperature (Janson et al. 2009). Based on the new combined constraint in this work, the orbit of $\epsilon$ Indi A b with respect to $\epsilon$ Indi A is shown in Fig. 6. It is apparent that the current separation of the planet from the star is optimal for direct imaging. The separation will increase from 1.1 as in 2020 to 3.3 as in 2030 and thus provides a perfect chance for imaging of a nearby cold Jupiter by JWST (Krist et al. 2007) or WFIRST (Melchior, Spergel 
Table 2. Parameters for $\epsilon$ Indi A and A b are taken from Gaia DR2 (Gaia Collaboration et al. 2018) or determined in this work. Except for rotation period and magnetic cycle for parameters determined in this work, the optimal value is estimated at the maximum a posteriori and the uncertainty interval is determined by the $10 \%$ and $90 \%$ quantiles of the posterior samples drawn by the MCMC chains.

\begin{tabular}{|c|c|c|c|}
\hline Parameter & Unit & Meaning & Value \\
\hline$m_{s}$ & $M_{\odot}$ & Stellar mass & $0.754_{-0.043}^{+0.043}$ \\
\hline$L_{s}$ & $L_{\odot}$ & Stellar Luminosity & $0.239_{-0.001}^{+0.001}$ \\
\hline$\alpha$ & degree & Right ascension & 330.87 \\
\hline$\delta$ & degree & Declination & -56.80 \\
\hline$\tilde{\omega}$ & mas & Parallax & $274.8_{-0.25}^{+0.25}$ \\
\hline$\mu_{\alpha}$ & $\operatorname{mas} / \mathrm{yr}$ & Proper motion in right ascension & $3967.04_{-0.38}^{+0.38}$ \\
\hline$\mu_{\delta}$ & $\operatorname{mas} / \mathrm{yr}$ & Proper motion in right ascension & $-2535.76_{-0.41}^{+0.41}$ \\
\hline$v_{r}$ & $\mathrm{~km} / \mathrm{s}$ & Systematic radial velocity & $-40.50_{-0.23}^{+0.23}$ \\
\hline$P_{\text {rot }}$ & day & Stellar rotation period & $-35.732_{-0.003}^{+0.006}$ \\
\hline$P_{\text {mag }}$ & day & Stellar magnetic cycle & $2500 \sim 3000$ \\
\hline$T_{\text {age }}$ & Gyr & Stellar age & $3.7 \sim 5.7$ \\
\hline$m_{p}$ & $M_{\text {Jup }}$ & Planet mass & $3.25_{-0.65}^{+0.39}$ \\
\hline$P$ & year & Orbital period & $45.20_{-4.77}^{+5.74}$ \\
\hline$a$ & $\mathrm{au}$ & Semi-major axis & $11.55_{-0.86}^{+0.98}$ \\
\hline$K$ & $\mathrm{~m} / \mathrm{s}$ & RV semi-amplitude & $29.22_{-6.07}^{+5.05}$ \\
\hline$e$ & & Eccentricity & $0.26_{-0.03}^{+0.07}$ \\
\hline$I$ & degree & Inclination & $64.25_{-6.09}^{+13.80}$ \\
\hline$\Omega$ & degree & Longitude of ascending node & $250.20_{-14.84}^{+14.72}$ \\
\hline$\omega$ & degree & Argument of periastron & $77.83_{-31.51}^{+20.21}$ \\
\hline$M_{0}$ & degree & Mean anomaly at reference epoch & $143.8_{-58.75}^{+23.38}$ \\
\hline$T_{0}$ & BJD & Reference epoch & 2448929.56 \\
\hline$T_{p}$ & BJD & Epoch at periastron & $2442332.95_{-3450.17}^{+2353.42}$ \\
\hline$b^{\mathrm{LC}}$ & $\mathrm{m} / \mathrm{s}$ & LC offset & $-16.60_{-5.10}^{+4.33}$ \\
\hline$b^{\mathrm{VLC}}$ & $\mathrm{m} / \mathrm{s}$ & VLC offset & $-22.97_{-3.84}^{+5.33}$ \\
\hline$b^{\text {HARPSpre }}$ & $\mathrm{m} / \mathrm{s}$ & HARPSpre offset & $-27.87_{-4.08}^{+5.16}$ \\
\hline$b^{\text {HARPSpost }}$ & $\mathrm{m} / \mathrm{s}$ & HARPSpost offset & $-14.23_{-5.75}^{+4.07}$ \\
\hline$b^{\mathrm{UVES}}$ & $\mathrm{m} / \mathrm{s}$ & UVES offset & $-15.04_{-4.09}^{+5.03}$ \\
\hline$\sigma^{\mathrm{LC}}$ & $\mathrm{m} / \mathrm{s}$ & LC RV jitter & $4.05_{-1.97}^{+3.01}$ \\
\hline$\sigma^{\mathrm{VLC}}$ & $\mathrm{m} / \mathrm{s}$ & VLC RV jitter & $0.42_{-0.15}^{+2.11}$ \\
\hline$\sigma^{\text {HARPSpre }}$ & $\mathrm{m} / \mathrm{s}$ & HARPSpre RV jitter & $1.45_{-0.05}^{+0.09}$ \\
\hline$\sigma^{\text {HARPSpost }}$ & $\mathrm{m} / \mathrm{s}$ & HARPSpost RV jitter & $1.02_{-0.07}^{+0.02}$ \\
\hline$\sigma^{\mathrm{UVES}}$ & $\mathrm{m} / \mathrm{s}$ & UVES RV jitter & $0.64_{-0.14}^{+0.09}$ \\
\hline$w_{1}^{\text {HARPSpre }}$ & $\mathrm{m} / \mathrm{s}$ & Amplitude of MA(2) for HARPSpre & $0.69_{-0.04}^{+0.07}$ \\
\hline$w_{2}^{\text {HARPSpre }}$ & $\mathrm{m} / \mathrm{s}$ & Amplitude of MA(2) for HARPSpre & $0.31_{-0.07}^{+0.06}$ \\
\hline $\ln \frac{\tau^{\text {HARPSpre }}}{1 \text { day }}$ & & Logarithmic MA time scale & $0.98_{-0.18}^{+0.24}$ \\
\hline$w_{1}^{\text {HARPSpost }}$ & $\mathrm{m} / \mathrm{s}$ & Amplitude of MA(1) for HARPSpost & $0.98_{-0.11}^{+0.00}$ \\
\hline $\ln \frac{\tau_{\text {HARPSpost }}}{1 \text { day }}$ & & Logarithmic MA time scale & $2.16_{-0.41}^{+4.22}$ \\
\hline$J_{\text {hip }}$ & & Hipparcos relative jitter & $0.26_{-0.22}^{+0.08}$ \\
\hline$J_{\text {gaia }}$ & & Gaia relative jitter & $0.19_{-0.14}^{+0.21}$ \\
\hline$\Delta \alpha$ & mas & Offset in $\alpha$ & $-0.99_{-0.09}^{+0.40}$ \\
\hline$\Delta \delta$ & mas & Offset in $\delta$ & $-0.01_{-0.26}^{+0.25}$ \\
\hline$\Delta \mu_{\alpha}$ & mas & Offset in $\mu_{\alpha}$ & $0.92_{-0.20}^{+0.29}$ \\
\hline$\Delta \mu_{\delta}$ & mas & Offset in $\mu_{\delta}$ & $0.34_{-0.30}^{+0.54}$ \\
\hline
\end{tabular}




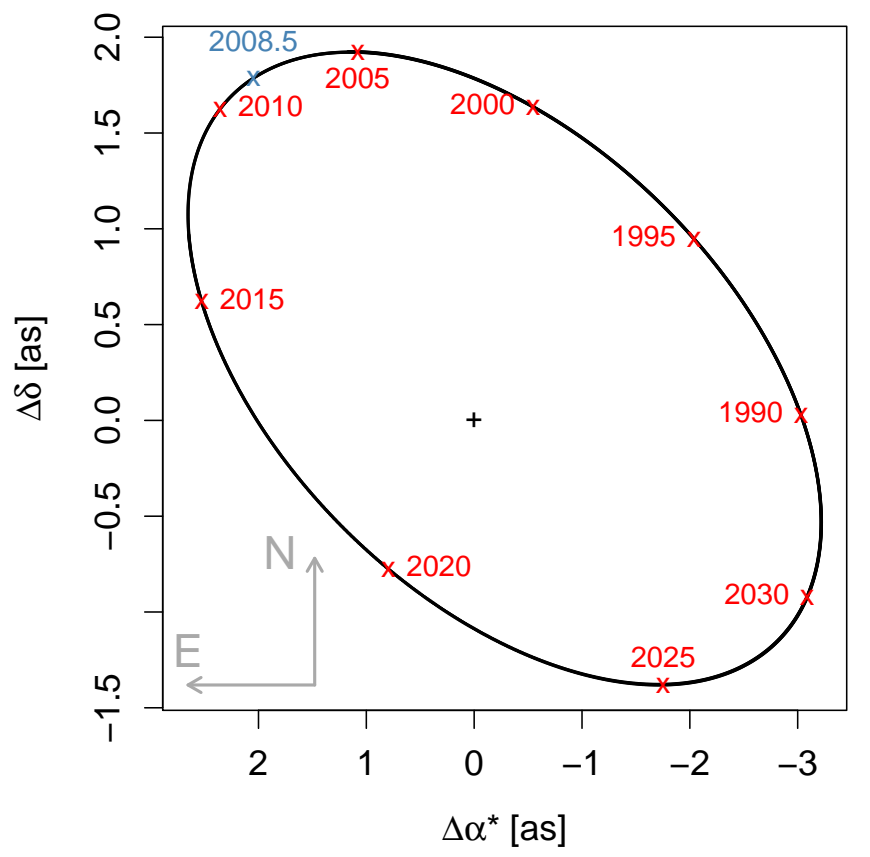

Figure 6. Orbit of $\epsilon$ Indi A b relative to its host star projected onto the sky plane. The position at different epochs are given as references for direct imaging. The separation between the planet and the star is about 1.1 as in 2020 . The light blue cross indicates the average epoch of the direc imaging data used by Janson et al. (2009). The North (N) and East (E) directions are shown by grey arrows. The black cross indicates the location of the star. Note that the orbit is shown in offset coordinates and $\Delta \alpha * \equiv \Delta \alpha \cos \delta$.

\& Lanz 2018). Althoug the separation between $\epsilon$ Indi Ab and its host star at the epochs of previous direct imaging (Janson et al. 2009) is wider than the one in 2020, our new constraint of the orbital parameters and mass of the planet can be used to optimize the observation strategy. Moreover, $\epsilon$ Indi A b would change its host star's position by about 1 mas during Gaia's five year mission and thus is detectable by Gaia with its $\sim 20 \mu$ as astrometric precision (Perryman et al. 2014).

\subsection{Dynamical stability of $\epsilon$ Indi A b}

With one Jovian planet and a binary brown dwarf system, the $\epsilon$ Indi system provides a benchmark case to test theories on the formation of giant planets and brown dwarfs. The age of the system is about 0.8 2.0 Gyr based on the estimated literature rotation period of 22 day determined from Ca II measurements. The kinematics of the system show an older age of $>9.87 \mathrm{Gyr}$ (Eker et al. 2015). Our analysis of the HARPSpre data set gives a rotation period of $35.7 \mathrm{~d}$ leading to an age of $3.7 \sim 5.7$ Gyr based on the rotation-age relationship given by Eker et al. (2015) which is more consistent with the age of 3.7 4.3 Gyr estimated by Scholz et al. (2003) based on an evolutionary model of the brown dwarf binary Ba and Bb. Thus we conclude that the age of the system is about $4 \mathrm{Gyr}$.

However, stars less massive than the Sun are unlikely to form more than one giant planet according to Kennedy \& Kenyon (2008). To investigate whether B a and B b were captured by $\epsilon$ Indi A either in or out of its birth environment, we calculate the escape radius derived by Feng \& Jones (2018) based on simulations of perturbations from stellar encounters on wide binaries and an encounter rate of $80 \mathrm{Myr}^{-1}$ for stellar encounters with periapsis less than 1 pc. The escape radius of $\epsilon$ Indi A is about $5600 \mathrm{au}$ which is larger than the projected separation (1459 au) between $\epsilon$ Indi B and A (Scholz et al. 2003). However, if $\epsilon$ Indi A migrated outward to its current location or captured $\epsilon$ Indi B during its formation, the encounter rate would be much higher and the escaped radius could be within the orbit of $\epsilon$ Indi B, which may also be much larger than the projected separation due to geometric effects. On the other hand, the brown dwarf binary may have been on a tighter orbit around the primary during the early evolution of the system and have migrated to its current orbit due to perturbations from the Galactic tide and stellar encounters.

Considering these scenarios of dynamical history and that the components of the $\epsilon$ Indi system are likely to have the same age, we conclude that the system was probably formed together and the brown dwarf binary has migrated from a tighter orbit to its current wide orbit. Such a migration would significantly influence the habitability of the system through periodic perturbations from the binary on any potentially habitable planets in the system. 


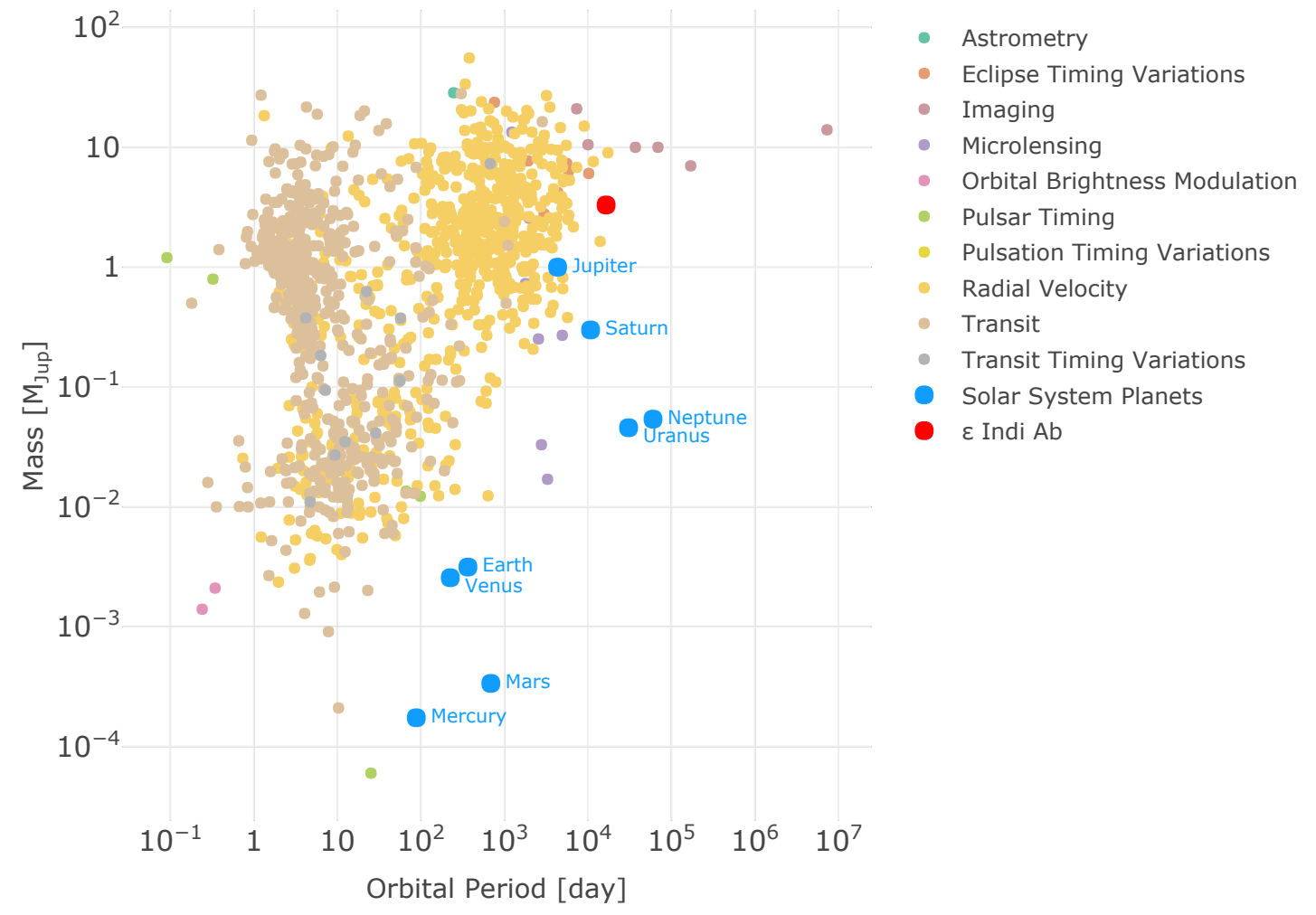

Figure 7. Scatter plot of orbital period and planetary mass for confirmed exoplanets, Solar System planets, and $\epsilon$ Indi A b. The Solar System planets are denoted by their names. The exoplanets are downloaded from NASA Exoplanet Archive and are color-coded for different detection methods.

\subsection{Comparing $\epsilon$ Indi A b with known exoplanets}

The discovery of the nearby Jupiter analog $\epsilon$ Indi A b is a milestone for the studies of the formation and evolution of Jupiter analogs. In Fig. 7, we show the mass and period of $\epsilon$ Indi A b compared with known exoplanets and the Solar System planets. We see that a few Jupiter-like planets (with 0.5 5 Jupiter mass) have been detected previously by the RV method though in these cases only provide minimum masses. So far none of them are confirmed or characterized by other methods. Although direct imaging is able to detect super-Jupiters and brown dwarfs around young stars, it is not able to probe the region below 8 Jupiter mass especially for evolved systems where Jupiter-like planets become very faint. Given the limitations of various methods in detecting wide-orbit gas giants, the detection of $\epsilon$ Indi A b by two methods illustrates how a good constraint (e.g., Fig. B1 can be set on a wide-orbit cold gas giant in an unpopulated region of exoplanet phase space by using astrometry and RV together.

\section{DISCUSSIONS AND CONCLUSION}

We analyze the RV and astrometry data of $\epsilon$ Indi A in the Agatha framework in combination with Bayesian methods. We confirm the suspected planet $\epsilon$ Indi A b to be a cold Jovian planet on a 11.55 au-wide orbit with an orbital period of 45.20 yr, making it the planet with a very long period from exoplanets detected by the RV methods. $\epsilon$ Indi A b is only 3.62 pc away from the Sun and is the closest Jovian exoplanet from the Earth. Given its proximity to the Sun, $\epsilon$ Indi A b is separated from its host star by as much as 3.5" and thus can be observed through direct imaging and astrometry, for example by JWST and Gaia (Krist et al. 2007; Perryman et al. 2014) though based on the Baraffe et al. (2003) isochrones it is likely to be too faint for the current ground-based imaging systems (e.g., Janson et al. 2009).

We also diagnose the other signals by comparing the BFPs for various RV data sets, noise models, and noise proxies. We find three signals at periods of about 11, 18 and $278 \mathrm{~d}$ in the RV data of $\epsilon$ Indi A. These signals are significant and can be constrained by Bayesian posterior samplings. Nevertheless, they are unlikely to be Keplerian because significant powers around these signals are found in the periodograms of various noise proxies especially in sodium lines. Based on the activity diagnostics, we conclude that all these signals together with the $2500 \mathrm{~d}$ signal in many noise proxies are caused by stellar 
activity. In particular, the 2500 and $278 \mathrm{~d}$ signals correspond to the magnetic cycles of $\epsilon$ Indi $\mathrm{A}$ while the 35,18 and $11 \mathrm{~d}$ signals are related to the stellar rotation. We find that the correlation between RVs and activity indicators depends on activity time scales. Thus the inclusion of them in the fit would remove activity-induced noise as well as introduce activity-induced signals.

The lack of signals with $K>1 \mathrm{~m} / \mathrm{s}$ and $P<4000$ days suggests a lack of super-Earths or mini-Neptunes in the habitable zone of $\epsilon$ Indi A which is 0.47 to 0.86 au according to (Kopparapu et al. 2014). Hence only Earth-like or smaller planets are allowed in the habitable zone. If these rocky planets are detected, $\epsilon$ Indi A would be similar to the Solar System, with close-in rocky planets and longer period gas giants. Thus, we also investigate the dynamical stability of this system according to the metrics of the stability of wide binaries under the perturbation of stellar encounters and Galactic tide (Feng \& Jones 2018). We find a considerable possibility that the brown dwarf binary in this system, $\epsilon$ Indi B a and B b, is unstable if they are on their current wide orbit. Hence the brown dwarf binary may have migrated from a tighter orbit to their current wide orbit, which might significantly influence the habitability of potentially Earth-like planet in the system.

Our successful detection and characterization of $\epsilon$ Indi A b through combined RV and astrometry analysis provides a benchmark example for the use of the astrometric difference between Gaia and Hipparcos data in characterizing massive planets. We find that the position offsets are more sensitive to nonlinear motion than the proper motion offsets. Although there are only two astrometry epochs, each epoch corresponds to four independent data points. Thus we are able to constrain the orbital parameters that the RV data is not sensitive to. We eagerly anticipate joint application of astrometry and RV methods in exoplanet detection and characterization with the epoch data in future Gaia data releases. $\epsilon$ Indi A b is optimal for direct imaging with a current spearation from its host of about 1 as. The separation will increase to 3.3 as in the coming decade, making it the nearest Jupiter analog for direct imaging by JWST or WFIRST.

\section{ACKNOWLEDGEMENTS}

We used the ESO Science Archive Facility to collect radial velocity data. We are very grateful to the referee for comments which significantly clarified the presentation of the results and the quality of the manuscript.

\section{APPENDIX A: POSTERIOR DISTRIBUTION OF MODEL PARAMETERS \\ APPENDIX B: POSTERIOR DISTRIBUTION OF MASS AND PERIOD \\ APPENDIX C: UVES RV DATA \\ REFERENCES}

Anglada-Escudé G. et al., 2016, Nat., 536, 437

Anglada-Escudé G., Butler R. P., 2012, ApJS, 200, 15

Baraffe I. et al., 2003, A\&A, 402, 701

Brandt T. D., 2018, ApJS, 239, 31

Calissendorff P., Janson M., 2018, A\&A, 615, A149

Demory B.-O. et al., 2009, A\&A, 505, 205

Eker Z. et al., 2015, AJ, 149, 131

Endl M. et al., 2002, A\&A, 392, 671

Feng F., Jones H. R. A., 2018, MNRAS, 474, 4412

Feng F., Jones H. R. A., Tuomi M., 2018, Research Notes of the American Astronomical Society, 2, 23

Feng F., Tuomi M., Jones H. R. A., 2017a, MNRAS, 470, 4794

Feng F., Tuomi M., Jones H. R. A., 2017b, A\&A, 605, A103

Feng F. et al., 2017, AJ, 154, 135

Feng F. et al., 2016, MNRAS, 461, 2440

Gaia Collaboration et al., 2018, A\&A, 616, A1

Gillon M. et al., 2016, Nature, 533, 221

Haario H. et al., 2006, Statistics and Computing, 16, 339

Janson M. et al., 2009, MNRAS, 399, 377

Jurić M., Tremaine S., 2008, ApJ, 686, 603

Kane S. R. et al., 2012, MNRAS, 425, 757

Kennedy G. M., Kenyon S. J., 2008, ApJ, 673, 502

Kervella P. et al., 2019, A\&A, 623, A72

Kipping D. M., 2013, MNRAS, 434, L51 


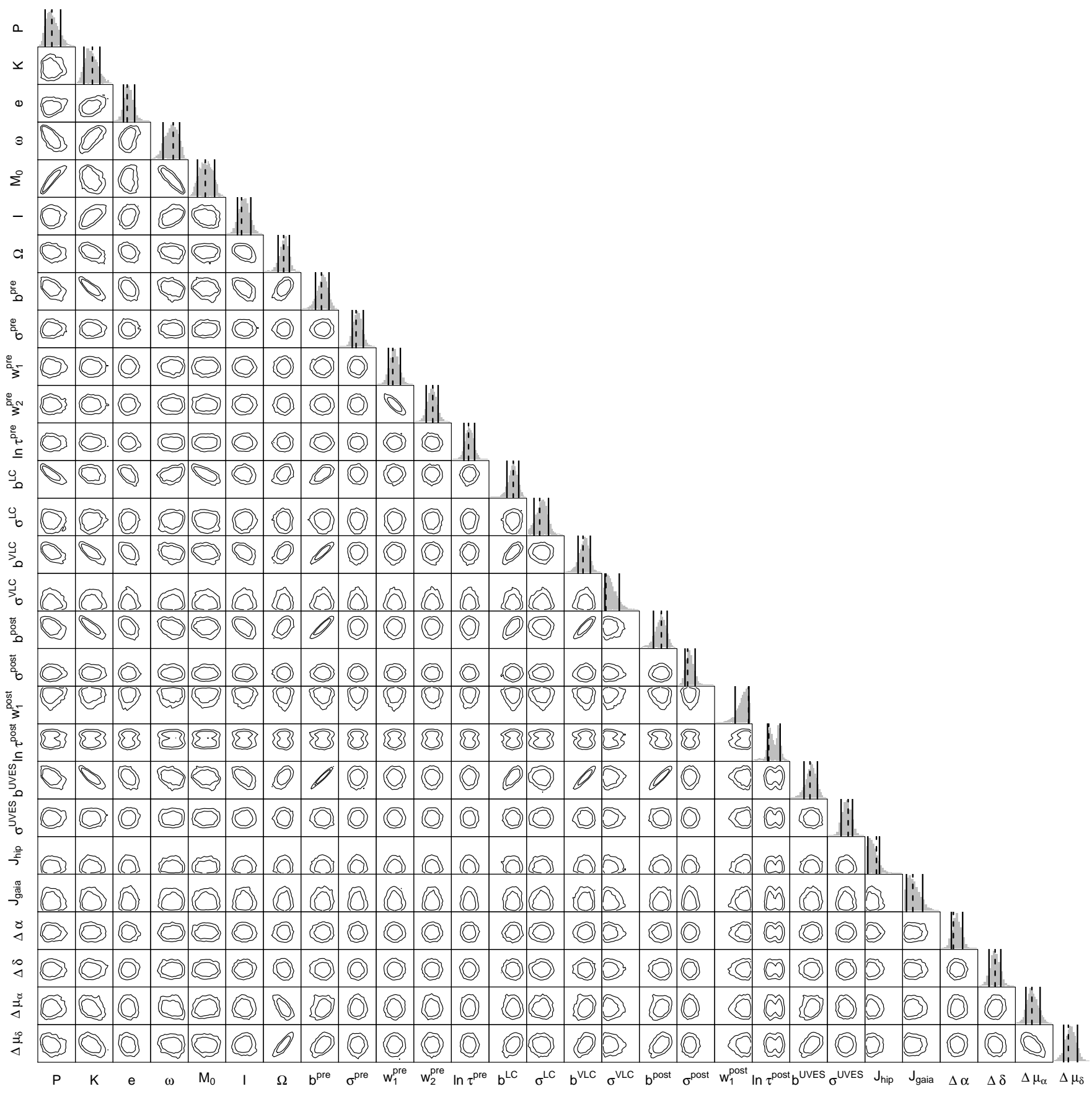

Figure A1. Two-dimensional posterior distributions for all model parameters. The first five parameters are for the Keplerian signal, $b$ is offset, $\omega$ and $\ln \tau$ are respectively the amplitude and logarithmic time scale of the MA model. The names of data sets are denoted by the superscripts for relevant parameters. The "pre" and "post" superscripts denote the HARPSpre and HARPSpost sets, respectively. The contours show the $68 \%$ and $90 \%$ confidence levels. The dashed lines denote the MAP parameter values in the posterior distribution while the solid lines show the $10 \%$ and $90 \%$ quantiles of the distribution.

Kopparapu R. K. et al., 2014, ApJL, 787, L29

Krist J. E. et al., 2007, in Proc. SPIE, Vol. 6693, Techniques and Instrumentation for Detection of Exoplanets III, p. 66930H

Kuzuhara M. et al., 2013, ApJ, 774, 11

Lindegren L. et al., 2018, A\&A, 616, A2

Lo Curto G. et al., 2015, The Messenger, 162, 9

Melchior P., Spergel D., Lanz A., 2018, AJ, 155, 102 


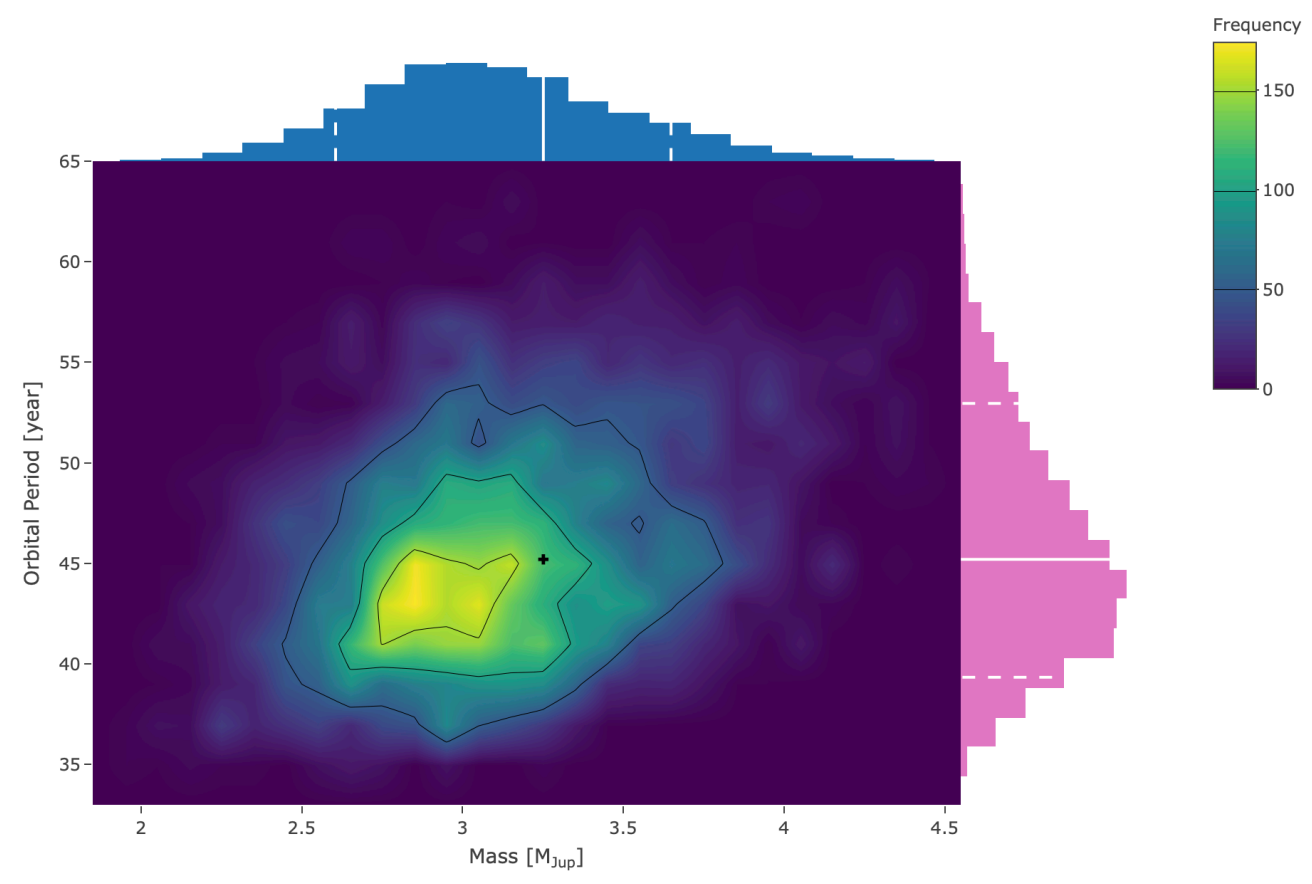

Figure B1. Posterior distribution of the mass and orbital period of $\epsilon$ Indi A b. The contour is calculated from a subsample drawn by the MCMC chains from the posterior distribution. Three contour levels corresponding to one, two and three sigma levels are shown by black lines. The MAP value (white solid lines) and the $10 \%$ and $90 \%$ quantiles (white dashed lines) of the orbital period and planetary mass are shown in the histograms of the mass and orbital period. The black cross in the contour denotes the MAP solution. This MAP solution differs from the mean solution because the distributions of the mass and orbital parameters are not Gaussian and are asymmetric.

Perryman M. et al., 2014, ApJ, 797, 14

Saar S. H., Osten R. A., 1997, MNRAS, 284, 803

Scholz R.-D. et al., 2003, A\&A, 398, L29

Schwarz G., et al., 1978, The annals of statistics, 6, 461

Snellen I. A. G., Brown A. G. A., 2018, Nature Astronomy, 2, 883

Tuomi M. et al., 2013, A\&A, 551, A79

Van Eylen V. et al., 2019, AJ, 157, 61

van Leeuwen F., 2007, A\&A, 474, 653

Wittenmyer R. A. et al., 2016, ApJ, 819, 28

Zechmeister M. et al., 2013, A\&A, 552, A78 
Table C1. UVES RV data.

\begin{tabular}{|c|c|c|}
\hline $\begin{array}{l}\text { BJD } \\
\text { (day) }\end{array}$ & $\begin{array}{l}\mathrm{RV} \\
(\mathrm{m} / \mathrm{s})\end{array}$ & $\begin{array}{c}\text { RV error } \\
(\mathrm{m} / \mathrm{s})\end{array}$ \\
\hline 2453272.49027 & -0.09 & 0.85 \\
\hline 2453272.4912 & -1.57 & 0.89 \\
\hline 2453272.49202 & 0.65 & 0.81 \\
\hline 2453272.49285 & 1.32 & 0.74 \\
\hline 2453272.49369 & -1.6 & 0.87 \\
\hline 2453272.49452 & -0.8 & 0.79 \\
\hline 2453272.49536 & 0.4 & 0.88 \\
\hline 2453272.49619 & 0.85 & 0.96 \\
\hline 2453272.49701 & -0.23 & 0.84 \\
\hline 2453272.49783 & -1.44 & 0.89 \\
\hline 2453272.49866 & -1.4 & 0.91 \\
\hline 2453272.49983 & -5.57 & 1.04 \\
\hline 2453272.50075 & -0.36 & 0.75 \\
\hline 2453272.50152 & -0.57 & 0.75 \\
\hline 2453272.50253 & -1.15 & 0.7 \\
\hline 2453272.5033 & -0.34 & 0.76 \\
\hline 2453272.50408 & -1.3 & 0.83 \\
\hline 2453272.50578 & -2.58 & 0.63 \\
\hline 2453272.50657 & -1.21 & 0.58 \\
\hline 2453272.5074 & -1.55 & 0.58 \\
\hline 2453272.50831 & -0.68 & 0.59 \\
\hline 2453272.50916 & -0.12 & 0.57 \\
\hline 2453272.50999 & 0.4 & 0.61 \\
\hline 2453272.51084 & -0.79 & 0.65 \\
\hline 2453272.51165 & -1.43 & 0.65 \\
\hline 2453272.51249 & -0.94 & 0.62 \\
\hline 2453272.51329 & 0.48 & 0.67 \\
\hline 2453272.51519 & -0.96 & 0.64 \\
\hline 2453272.51597 & -0.66 & 0.61 \\
\hline 2453272.51676 & -1.59 & 0.6 \\
\hline 2453272.51754 & -1.25 & 0.6 \\
\hline 2453272.5183 & -2.49 & 0.61 \\
\hline 2453272.51908 & -0.95 & 0.58 \\
\hline 2453272.51983 & -0.43 & 0.64 \\
\hline 2453272.52057 & -1.63 & 0.62 \\
\hline 2453272.5213 & -0.59 & 0.64 \\
\hline 2453272.52203 & -3.72 & 0.66 \\
\hline 2453272.52391 & -1.05 & 0.7 \\
\hline 2453272.52471 & -1.57 & 0.66 \\
\hline 2453272.5255 & -0.93 & 0.67 \\
\hline 2453272.5263 & -0.07 & 0.67 \\
\hline 2453272.52711 & -1.36 & 0.65 \\
\hline 2453272.52792 & -1.95 & 0.66 \\
\hline 2453272.52869 & -1.58 & 0.64 \\
\hline 2453272.52947 & -1.18 & 0.71 \\
\hline 2453272.53024 & -1.39 & 0.6 \\
\hline 2453272.53101 & -0.96 & 0.67 \\
\hline 2453272.53286 & 0 & 0.77 \\
\hline 2453272.5336 & -2.54 & 0.8 \\
\hline 2453272.53432 & -1.16 & 0.77 \\
\hline 2453272.53505 & -0.83 & 0.79 \\
\hline 2453272.53917 & 0.09 & 1.01 \\
\hline 2453272.53987 & -0.25 & 0.91 \\
\hline
\end{tabular}




\section{F. Feng et al.}

Table C2. Table C1 continue.

\begin{tabular}{ccc}
\hline \hline BJD & RV & RV error \\
(day) & $(\mathrm{m} / \mathrm{s})$ & $(\mathrm{m} / \mathrm{s})$ \\
\hline 2453272.54076 & -0.48 & 0.83 \\
2453272.54151 & -0.47 & 0.65 \\
2453272.54228 & -0.94 & 0.69 \\
2453272.54304 & -0.24 & 0.69 \\
2453272.54382 & 0.26 & 0.63 \\
2453272.54464 & -1.43 & 0.64 \\
2453272.54546 & -0.25 & 0.66 \\
2453272.54626 & 0.3 & 0.62 \\
2453272.54706 & -2.44 & 0.67 \\
2453272.54782 & -0.13 & 0.72 \\
2453272.55005 & -0.15 & 0.8 \\
2453272.55081 & -1.1 & 0.61 \\
2453272.55158 & -1.15 & 0.63 \\
2453272.55236 & 0.1 & 0.66 \\
2453272.55314 & -0.19 & 0.63 \\
2453272.55393 & -1.02 & 0.62 \\
2453272.55473 & -1.84 & 0.56 \\
2453272.55552 & -2.06 & 0.71 \\
2453272.55632 & -0.74 & 0.62 \\
2453272.55718 & -2.83 & 0.61 \\
2453272.55823 & -1.37 & 0.56 \\
2453272.55997 & -1.39 & 0.62 \\
2453272.56082 & -2.43 & 0.63 \\
2453272.56168 & -0.22 & 0.64 \\
2453272.56255 & -1.36 & 0.59 \\
2453272.56337 & -0.14 & 0.69 \\
2453272.56418 & -3.49 & 0.7 \\
2453272.56499 & -0.85 & 0.68 \\
2453272.5658 & -0.62 & 0.68 \\
2453272.56662 & -1.93 & 0.61 \\
2453272.56745 & -0.03 & 0.64 \\
2453272.56828 & -2.14 & 0.62 \\
2453272.5702 & -2.37 & 1.13 \\
2453272.57094 & 0.26 & 0.77 \\
2453272.57173 & -1.13 & 0.65 \\
2453272.57254 & -1.59 & 0.67 \\
2453272.57334 & -1.85 & 0.67 \\
2453272.57413 & 0.06 & 0.73 \\
2453272.57492 & -2.69 & 0.69 \\
2453272.57572 & -0.67 & 0.67 \\
2453272.57652 & -1.71 & 0.69 \\
2453272.57733 & -1.24 & 0.7 \\
2457905.83292 & 7.65 & 0.93 \\
2457905.839 & 6.92 & 0.94 \\
2457905.8412 & 7.45 & 0.93 \\
2457905.84344 & 5.56 & 0.86 \\
2457905.84566 & 7.99 & 0.88 \\
2457905.86181 & 6.97 & 0.93 \\
2457905.86267 & 8.5 & 0.95 \\
2457905.86353 & 9.13 & 0.93 \\
2457905.86439 & 8.53 & 0.96 \\
2457905.86689 & 7.93 & 0.98 \\
2457905.86774 & 8.08 & 0.96 \\
2457905.86861 & 7.65 & 0.96 \\
2457905.86947 & 6.17 & 1.01 \\
2457905.8727 & 8.67 & 0.92 \\
\hline & 9.02 & 0.91 \\
\hline
\end{tabular}


Table C3. Table C1 continue.

\begin{tabular}{|c|c|c|}
\hline $\begin{array}{l}\text { BJD } \\
\text { (day) }\end{array}$ & $\begin{array}{c}\mathrm{RV} \\
(\mathrm{m} / \mathrm{s})\end{array}$ & $\begin{array}{c}\text { RV error } \\
(\mathrm{m} / \mathrm{s})\end{array}$ \\
\hline 2457905.87356 & 7.78 & 0.95 \\
\hline 2457905.87442 & 8.69 & 0.92 \\
\hline 2457905.8768 & 7.64 & 0.98 \\
\hline 2457905.87766 & 6.51 & 0.95 \\
\hline 2457905.87852 & 6.94 & 0.89 \\
\hline 2457905.87939 & 8.87 & 0.9 \\
\hline 2457905.88175 & 9.46 & 0.92 \\
\hline 2457905.88262 & 8.36 & 0.94 \\
\hline 2457905.88347 & 7.95 & 0.92 \\
\hline 2457905.88433 & 7.99 & 0.9 \\
\hline 2457905.8868 & 6.58 & 1.02 \\
\hline 2457905.88765 & 6.43 & 0.94 \\
\hline 2457905.88852 & 7.75 & 1.04 \\
\hline 2457905.88938 & 5.49 & 0.97 \\
\hline 2457905.89185 & 7.1 & 0.95 \\
\hline 2457905.89271 & 10.2 & 0.94 \\
\hline 2457905.89357 & 8.43 & 0.95 \\
\hline 2457905.89443 & 7 & 0.96 \\
\hline 2457905.8969 & 8.72 & 0.96 \\
\hline 2457905.89775 & 8.33 & 0.91 \\
\hline 2457905.89861 & 7.88 & 0.97 \\
\hline 2457905.89948 & 7.74 & 0.93 \\
\hline 2457993.67726 & 5.97 & 0.84 \\
\hline 2457993.67812 & 6.95 & 0.91 \\
\hline 2457993.67898 & 7.09 & 0.86 \\
\hline 2457993.67984 & 7.72 & 0.87 \\
\hline 2457993.68162 & 7.57 & 0.86 \\
\hline 2457993.68247 & 7.71 & 0.93 \\
\hline 2457993.68334 & 8.27 & 0.94 \\
\hline 2457993.6842 & 8.63 & 0.89 \\
\hline 2457993.68594 & 7.33 & 0.88 \\
\hline 2457993.68679 & 6.02 & 0.96 \\
\hline 2457993.68765 & 8.74 & 0.92 \\
\hline 2457993.68851 & 6.31 & 0.89 \\
\hline 2457993.69038 & 6.71 & 0.92 \\
\hline 2457993.69124 & 7.19 & 0.91 \\
\hline 2457993.6921 & 8.14 & 0.87 \\
\hline 2457993.69296 & 6.28 & 0.95 \\
\hline 2457993.69527 & 6.47 & 0.93 \\
\hline 2457993.69613 & 4.92 & 0.9 \\
\hline 2457993.69699 & 6.77 & 0.98 \\
\hline 2457993.69786 & 7.28 & 0.93 \\
\hline 2457993.69971 & 7.86 & 0.9 \\
\hline 2457993.70057 & 7.29 & 0.87 \\
\hline 2457993.70144 & 7.69 & 0.82 \\
\hline 2457993.70229 & 7.28 & 0.88 \\
\hline 2457993.70522 & 6.38 & 0.89 \\
\hline 2457993.70608 & 7.32 & 0.93 \\
\hline 2457993.70693 & 5.77 & 0.88 \\
\hline 2457993.7078 & 6.36 & 0.96 \\
\hline 2457993.70968 & 6.6 & 0.87 \\
\hline 2457993.71054 & 6.81 & 0.92 \\
\hline 2457993.7114 & 7.27 & 0.88 \\
\hline 2457993.71226 & 7.82 & 0.98 \\
\hline
\end{tabular}

